\title{
Non-local viscosity of polymer melts approaching their glassy state
}

Puscasu, Ruslan; Todd, Billy; Daivis, Peter; Hansen, Jesper Schmidt

Published in:

Journal of Chemical Physics

DOI:

$10.1063 / 1.3499745$

Publication date:

2010

Document Version

Publisher's PDF, also known as Version of record

Citation for published version (APA):

Puscasu, R., Todd, B., Daivis, P., \& Hansen, J. S. (2010). Non-local viscosity of polymer melts approaching their glassy state. Journal of Chemical Physics, 133(14). https://doi.org/10.1063/1.3499745

\section{General rights}

Copyright and moral rights for the publications made accessible in the public portal are retained by the authors and/or other copyright owners and it is a condition of accessing publications that users recognise and abide by the legal requirements associated with these rights.

- Users may download and print one copy of any publication from the public portal for the purpose of private study or research.

- You may not further distribute the material or use it for any profit-making activity or commercial gain.

- You may freely distribute the URL identifying the publication in the public portal.

\section{Take down policy}

If you believe that this document breaches copyright please contact rucforsk@kb.dk providing details, and we will remove access to the work immediately and investigate your claim. 


\title{
Nonlocal viscosity of polymer melts approaching their glassy state
}

\author{
R. M. Puscasu, ${ }^{1, a)}$ B. D. Todd, ${ }^{1, b)}$ P. J. Daivis, ${ }^{2, c)}$ and J. S. Hansen ${ }^{3, d)}$ \\ ${ }^{1}$ Centre for Molecular Simulation, Swinburne University of Technology, P.O. Box 218, Hawthorn, \\ Victoria 3122, Australia \\ ${ }^{2}$ Applied Physics, School of Applied Sciences, RMIT University, G.P.O. Box 2476, Melbourne, \\ Victoria 3001, Australia \\ ${ }^{3}$ DNRF Centre "Glass and Time", Department of Sciences, Roskilde University, \\ P.O. Box 260, DK-4000 Roskilde, Denmark
}

(Received 29 March 2010; accepted 20 September 2010; published online 11 October 2010)

\begin{abstract}
The nonlocal viscosity kernels of polymer melts have been determined by means of equilibrium molecular dynamics upon cooling toward the glass transition. Previous results for the temperature dependence of the self-diffusion coefficient and the value of the glass transition temperature are confirmed. We find that it is essential to include the attractive part of the interatomic potential in order to observe a strong glass transition. The width of the reciprocal space kernel decreases dramatically near the glass transition, being described by a deltalike function near and below the glass transition, leading to a very broad kernel in physical space. Thus, spatial nonlocality turns out to play an important role in polymeric fluids at temperatures near the glass transition temperature. (C) 2010 American Institute of Physics. [doi:10.1063/1.3499745]
\end{abstract}

\section{INTRODUCTION}

Polymeric materials are one of the most representative compounds that can exist in a glassy state and they have attracted significant interest over the last decade. Model polymer melts are convenient systems for computer simulations of materials in metastable equilibrium near the glass transition temperature. The dynamics of such melts are generally explained by various theories such as mode coupling theory, ${ }^{1}$ entropy theory, ${ }^{2}$ the free volume approach, ${ }^{3}$ or the spin model. ${ }^{4}$ A body of literature exists on the viscoelastic properties and the behavior of the viscosity near the glass transition. ${ }^{5-8}$ There is, however, much less information about the spatial nonlocality of the viscosity near the glass transition.

Newton's law of viscosity relates the shear stress in a deforming material at some point in space and time to the local strain rate via a constant viscosity. However, at the atomic and molecular length scales, a nonlocal constitutive relation allowing the viscosity to be a nonlocal property of a fluid in space and time must be employed. Such a relation can be expressed as ${ }^{9,10}$

$$
P_{x y}(\mathbf{r}, t)=-\int_{0}^{t} \int_{-\infty}^{\infty} \eta\left(\mathbf{r}-\mathbf{r}^{\prime}, t-t^{\prime}\right) \dot{\gamma}\left(\mathbf{r}^{\prime}, t^{\prime}\right) d \mathbf{r}^{\prime} d t^{\prime},
$$

where $P_{x y}(\mathbf{r}, t)$ is the $(x, y)$ off-diagonal component of the pressure tensor, $\dot{\gamma}\left(\mathbf{r}^{\prime}, t^{\prime}\right)$ is the shear strain rate, and $\eta(\mathbf{r}$ $\left.-\mathbf{r}^{\prime}, t-t^{\prime}\right)$ is the nonlocal viscosity kernel. Indeed, it has been shown that in all but the simplest flows (e.g., planar Couette and Poiseuille flow) the finite extent of the viscosity

\footnotetext{
${ }^{a)}$ Electronic mail: rpuscasu@swin.edu.au.

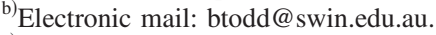

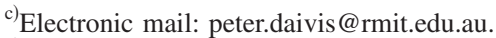

${ }^{\mathrm{d})}$ Electronic mail: jesperschmidthansen@gmail.com.
}

kernel can play a significant role when the strain rate varies significantly over molecular distance scales. ${ }^{11,12}$

Nonlocal viscosity kernels have been determined for atomic fluids ${ }^{13,14}$ and a few simple molecular fluids (chlorine, ${ }^{14}$ carbon dioxide, ${ }^{15}$ water, ${ }^{15-17}$ and polymer melts at room temperature ${ }^{18}$ ). However, to our knowledge, a similar study for the temperature dependence of the viscosity kernel of a liquid approaching its glassy state has not been carried out except for a few recent works by Furukawa and Tanaka ${ }^{19}$ and Kim and Keyes. ${ }^{20}$ Furukawa and Tanaka, ${ }^{19}$ for instance, studied a glass-forming binary mixture of simple atomic species and they confirmed the nonlocal nature of the viscous transport and linked it to the existence of dynamic heterogeneities. There are, however, a few key differences between our results and Furukawa and Tanaka's work. For example, we use more robust functions to fit the nonlocal viscosity data, a more extended set of stress and momentum density correlation function results, and we have analyzed both the reciprocal and the real space viscosity kernels (Furukawa and Tanaka only examined the reciprocal space kernels). Kim and Keyes ${ }^{20}$ also evaluated the wavevector dependent shear viscosity for a range of temperatures in a binary Lennard-Jones liquid system and observed a growing correlation length associated with a dynamically homogeneous domain. Isobe and Alder characterized the molasses tail (long time tail of the stress autocorrelation function) for a systems of elastic two-dimensional hard disks and argued that the enhanced viscosity near solidification is due to a transitory existence of solid nuclei. ${ }^{21}$

In our study we address this problem by computing the viscosity kernel of a polymer melt as it is cooled down toward its glass transition temperature. We must stress that the main objective is not a precise prediction of the glass transition, but rather an analysis of temperature dependence of the viscosity kernel (i.e., the shape of the kernel) as we approach 
$T_{g}$. Although a large number of publications exist on glass transition temperature estimation, it is often difficult to compare new work with published data because they have been computed for different systems (bulk liquids, films, networks), under different conditions (constant pressure, constant volume), by applying different models (potential energy functions, cutoff distances) for various chain lengths and densities. Therefore, we will present the results for selfdiffusion coefficients and stress autocorrelation functions which show the characteristics of the glassy state. We also estimate the value of $T_{g}$ and compare the values with previous work where possible.

Another contribution of this paper is the analysis of the chain length influence on the viscosity kernel. Although our previous results ${ }^{18}$ indicate little difference when increasing the chain length to up to 100 beads per chain at a dense fluid state point $(\rho=0.84, T=1.0)$, it is worth looking at much lower temperature regions which might offer an insight into where exactly the onset of large spatial correlations occurs.

This paper is structured as follows. Section II provides a conceptual background and, in particular, the expressions for the complex wave-vector and frequency dependent viscosity. In Sec. III we describe our model and simulation conditions. In Sec. IV we present the main results from equilibrium molecular dynamics (MD) simulations. In addition to the transverse momentum density autocorrelation function, stress autocorrelation functions, and wave-vector dependent viscosity, the self-diffusion calculations used for glass transition temperature estimation are also presented. The real space kernels and the structural scaling of the kernels are then given. Finally, we summarize and conclude our analysis in Sec. V.

\section{METHOD}

The approach used in this paper has been discussed in detail elsewhere ${ }^{18}$ so we only recall here the main results and relations required for the discussion that follows.

In principle, the wave-vector and frequency dependent viscosity could be evaluated by using two different expressions. One is in terms of the Fourier-Laplace transform of the transverse momentum density autocorrelation function (ACF), $C_{\perp}(\mathbf{k}, t)$, and one is in terms of the Fourier-Laplace transform of the stress tensor autocorrelation function, $N(\mathbf{k}, t) .{ }^{10}$ Both correlation functions can be defined either in atomic or molecular representation. However, we only consider the wave-vector and frequency dependent viscosities defined for the atomic representation. The molecular stress tensor is not symmetric instantaneously, hence the formulation of correlation function expressions is more complex.

We define the Fourier-Laplace transform (one-sided Fourier transform) as $\mathcal{L}[f(t)]=\widetilde{f}(\omega)=\int_{0}^{\infty} f(t) e^{-i \omega t} d t$. For the sake of simplicity of notation and consistency with the notation used in previous publications, we drop the tilde sign over the correlation functions and keep the tilde notation over the Fourier-Laplace transformed correlation functions only. If we set the wave-vector $\mathbf{k}=\left(0, k_{y}, 0\right)$ and let $J_{x}$ be the component of the momentum density in the $x$ direction, the expression for the wave-vector and frequency dependent viscosity in terms of $\widetilde{C}_{\perp}\left(k_{y}, \omega\right)$ takes the form ${ }^{10}$

$$
\widetilde{\eta}\left(k_{y}, \omega\right)=\frac{\rho}{k_{y}^{2}} \frac{C_{\perp}\left(k_{y}, t=0\right)-i \omega \widetilde{C}_{\perp}\left(k_{y}, \omega\right)}{\widetilde{C}_{\perp}\left(k_{y}, \omega\right)},
$$

where $\rho$ is the atomic mass density of the fluid and $\widetilde{C}_{\perp}\left(k_{y}, \omega\right)$ is the Laplace transform of the ensemble averaged transverse momentum density autocorrelation function $C_{\perp}\left(k_{y}, t\right)$, which is defined as

$$
C_{\perp}\left(k_{y}, t\right)=\frac{1}{V}\left\langle J_{x}^{*}\left(k_{y}, t\right) J_{x}\left(k_{y}, t=0\right)\right\rangle,
$$

where $V$ is the simulation volume. The integral of the transverse momentum density $\mathrm{ACF}$ is given as

$$
I_{C_{\perp}}\left(k_{y}, t\right)=\int_{0}^{t} C_{\perp}\left(k_{y}, t^{\prime}\right) d t^{\prime} .
$$

The Fourier transform of the momentum density in the atomic representation is

$$
\widetilde{\mathbf{J}}^{A}(\mathbf{k}, t)=\sum_{i=1}^{N_{m}} \sum_{\alpha=1}^{N_{s}} \mathbf{p}_{i \alpha}(t) e^{i \mathbf{k} \cdot \mathbf{r}_{i \alpha}},
$$

where the inner summation extends over the number of interaction sites $N_{s}$ in a molecule and the outer summation extends over the number of molecules $N_{m}$ in the system. For molecules composed of $N_{s}$ atoms, we can define the molecular mass as $M_{i}=\sum_{\alpha=1}^{N_{s}} m_{i \alpha}$, the position of the molecular center of mass as $\mathbf{r}_{i}=\sum_{\alpha=1}^{N_{s}} m_{i \alpha} \mathbf{r}_{i \alpha} / M_{i}$, and the momentum of the molecule as $\mathbf{p}_{i}=\sum_{\alpha=1}^{N_{S}} \mathbf{p}_{i \alpha}$. Thus, the Fourier transform of the molecular momentum density can be written as

$$
\widetilde{\mathbf{J}}^{M}(\mathbf{k}, t)=\sum_{i=1}^{N_{m}} \mathbf{p}_{i}(t) e^{i \mathbf{k} \cdot \mathbf{r}_{i}} .
$$

The zero time value of $C_{\perp}\left(k_{y}, t=0\right)$ for an atomic fluid in the thermodynamic limit is

$$
C_{\perp}\left(k_{y}, t=0\right)=\rho k_{B} T,
$$

where $k_{B}$ is Boltzmann's constant. The theoretical value of $C_{\perp}\left(k_{y}, t=0\right)=\rho k_{B} T$ for an atomic fluid obtained from a canonical ensemble average in the thermodynamic limit differs slightly from the theoretical value in the simulated isokinetic ensemble given by $C_{\perp}\left(k_{y}, t=0\right)=\rho k_{B} T\left(1-4 N_{s} N_{m} / 3\right)$ because the total peculiar (i.e., thermal) kinetic energy and three components of the momenta are constants of the motion in our simulations. For a molecular fluid with constrained bonds, we must also account for the internal bond constraints in deriving a relationship between the zero time value of the atomic momentum density $\mathrm{ACF}$ and the temperature. ${ }^{18}$ To ensure numerical consistency of our results, we use the simulated value of $C_{\perp}\left(k_{y}, t=0\right)$ rather than Eq. (7) in our calculations of the wavevector dependent viscosity.

The expression for the wave-vector and frequency dependent viscosity in terms of the ACF of the shear stress $N\left(k_{y}, t\right)$ takes the form

$$
\widetilde{\eta}\left(k_{y}, \omega\right)=\frac{\tilde{N}\left(k_{y}, \omega\right)}{C_{\perp}\left(k_{y}, t=0\right) / \rho k_{B} T-k^{2} \tilde{N}\left(k_{y}, \omega\right) / i \omega \rho},
$$

where the shear stress correlation function is defined as 


$$
N\left(k_{y}, t\right)=\frac{1}{V k_{B} T}\left\langle P_{x y}^{*}\left(k_{y}, t\right) P_{x y}\left(k_{y}, 0\right)\right\rangle
$$

and its Laplace transform as

$$
\tilde{N}\left(k_{y}, \omega\right)=\mathcal{L}\left[N\left(k_{y}, t\right)\right],
$$

respectively. The integral of the shear stress ACF can be written as

$$
I_{N}\left(k_{y}, t\right)=\int_{0}^{t} N\left(k_{y}, t^{\prime}\right) d t^{\prime}
$$

which converges to the Green-Kubo viscosity for zero wavevector and to zero at nonzero wave-vector. Therefore, Eq. (2) must be used when nonzero wave-vector viscosities are calculated. ${ }^{10}$ Again, we point out that Eq. (8) is only valid in the atomic representation.

The wave-vector dependent atomic pressure tensor for a polymer system composed of $N_{m}$ molecules and $N_{s}$ sites per molecule is defined as

$$
\begin{aligned}
\widetilde{\mathbf{P}}^{\mathrm{FJCFENE}}(\mathbf{k}, t)= & \sum_{i=1}^{N_{m}} \sum_{\alpha=1}^{N_{s}} \frac{\mathbf{p}_{i \alpha} \mathbf{p}_{i \alpha}}{m_{i \alpha}} e^{i \mathbf{k} \cdot \mathbf{r}_{i \alpha}}-\frac{1}{2} \sum_{i=1}^{N_{m}} \sum_{\alpha=1}^{N_{s}} \sum_{j \neq i}^{N_{m}} \sum_{\beta=1}^{N_{s}} \mathbf{r}_{i \alpha j \beta} \\
& \times \mathbf{F}_{i \alpha j \beta} g\left(i \mathbf{k} \cdot \mathbf{r}_{i \alpha j \beta}\right) e^{i \mathbf{k} \cdot \mathbf{r}_{i \alpha j \beta}} \\
& -\sum_{i=1}^{N_{m}} \sum_{\alpha=1}^{N_{s}-2} \sum_{\beta=\alpha+2}^{N_{s}} \mathbf{r}_{i \alpha i \beta} \mathbf{F}_{i \alpha i \beta} g\left(i \mathbf{k} \cdot \mathbf{r}_{i \alpha i \beta}\right) e^{i \mathbf{k} \cdot \mathbf{r}_{i \alpha i \beta}} \\
& +\sum_{i=1}^{N_{m}} \sum_{\alpha=1}^{N_{s}} \mathbf{r}_{i \alpha} \mathbf{F}_{i \alpha}^{C / \mathrm{FENE}} g\left(i \mathbf{k} \cdot \mathbf{r}_{i \alpha}\right) e^{i \mathbf{k} \cdot \mathbf{r}_{i \alpha}}
\end{aligned}
$$

$\mathbf{F}_{i \alpha j \beta}$ is the LJ force acting on site $\alpha$ of molecule $i$ due to site $\beta$ of molecule $j$ and $\mathbf{F}_{i \alpha}^{C / \mathrm{FENE}}$ is either the constraint force for freely jointed chains (FJCs) or the bond force for finite extensible nonlinear elastic chains (FENE) on site $\alpha$ of molecule $i . \mathbf{r}_{i \alpha j \beta}=\left(\mathbf{r}_{j \beta}-\mathbf{r}_{i \alpha}\right)_{\min }$ is the minimum image separation of site $\alpha$ of molecule $i$ from site $\beta$ of molecule $j . g(i \mathbf{k} \cdot \mathbf{r})$ are the Fourier transforms of the Irving-Kirkwood operators, ${ }^{22}$ where $g(x)=\left(e^{x}-1\right) / x=\sum_{n=0}^{\infty}(x)^{n} /(n+1)$ !, with $x=i \mathbf{k} \cdot \mathbf{r}_{i \alpha j \beta}$, $x=i \mathbf{k} \cdot \mathbf{r}_{i \alpha i \beta}$, and $x=i \mathbf{k} \cdot \mathbf{r}_{i \alpha}$, respectively. The pressure tensor in the molecular representation is defined as

$$
\widetilde{\mathbf{P}}^{M}(\mathbf{k}, t)=\sum_{i=1}^{N_{m}} \frac{\mathbf{p}_{i} \mathbf{p}_{i}}{M_{i}} e^{i \mathbf{k} \cdot \mathbf{r}_{i}}-\frac{1}{2} \sum_{i=1}^{N_{m}} \sum_{j \neq i}^{N_{m}} \mathbf{r}_{i j} \mathbf{F}_{i j}^{\mathrm{inter}} g\left(i \mathbf{k} \cdot \mathbf{r}_{i j}\right) e^{i \mathbf{k} \cdot \mathbf{r}_{i j},}
$$

where $\mathbf{F}_{i j}^{\mathrm{inter}}$ represents the intermolecular force. $\mathbf{r}_{i j}=\left(\mathbf{r}_{j}\right.$ $\left.-\mathbf{r}_{i}\right)_{\min }$ is the minimum image separation of the center of mass of molecule $i$ from the center of mass of molecule $j$. A more detailed discussion of momentum densities and pressure tensor in atomic and molecular formalisms can be found in Refs. 18, 23, and 24.

\section{SIMULATION}

\section{A. Model}

Our simulation methods have been described in detail in a previous publication. ${ }^{18}$ In brief, we employ a freely jointed tangent chain model (FJC) $)^{25,26}$ and the Kremer-Grest FENE chain model $^{27}$ to simulate three polymeric systems.

(i) In the first system each molecule consists of $N_{s}$ sites of equal mass $m_{i \alpha}$ interacting via a truncated $\left(r_{c}=2^{1 / 6}\right)$ and shifted Lennard-Jones (LJ) potential known as the Weeks-Chandler-Andersen (WCA) interaction, ${ }^{28}$

$$
\Phi_{i j}\left(r_{i j}\right)= \begin{cases}4 \epsilon\left[\left(\frac{\sigma}{r_{i j}}\right)^{12}-\left(\frac{\sigma}{r_{i j}}\right)^{6}\right]-\Phi_{c}, & r_{i j}<r_{c} \\ 0, & r_{i j} \geq r_{c},\end{cases}
$$

where $r_{i j}$ is the interatomic separation, $\epsilon$ is the potential well depth, and $\sigma$ is the value of $r_{i j}$ at which the unshifted potential is zero. The shift $\Phi_{c}$ is the value of the unshifted potential at the cutoff $r_{i j}=r_{c}$ and is introduced to eliminate the discontinuity in the potential energy. At distances greater than the cutoff distance $r_{c}$, the potential is zero. The sites are joined by rigidly constrained bonds of length $l=\sigma$. The WCA interactions in this model can occur between any two different sites except those connected by a bond. This case will be termed, for simplicity, the FJC-WCA system.

We note that all quantities in this paper are reported in terms of reduced units which are relative to the Lennard-Jones parameters. The conversion from real units to reduced units gives reduced length $\mathbf{r}^{*}$ $=\mathbf{r} / \sigma$, reduced density $\rho^{*}=\rho / \sigma^{3}$ reduced temperature $T^{*}=k_{B} T / \epsilon$, reduced time $t^{*}=t /\left(\sigma(m / \epsilon)^{1 / 2}\right.$, reduced pressure $\mathbf{P}^{*}=\mathbf{P}\left(\sigma^{3} / \epsilon\right)$, reduced energy $E^{*}=E / \epsilon$, and reduced viscosity $\eta^{*}=\eta \sqrt{\sigma^{4} /(m \epsilon)}$. For the remainder of this paper the asterisk is dropped.

(ii) In the second system we also include the attractive part of the shifted LJ interaction by letting $r_{c}=2.5 \sigma$. This case will be termed the FJC-LJ system.

(iii) In the third system, termed as FENE-LJ, in addition to the LJ potential, adjacent connected beads also interact through a finitely extensible nonlinear elastic potential,

$$
\Phi_{i j}^{\mathrm{FENE}}\left(r_{i j}\right)=\left\{\begin{array}{cc}
-\frac{1}{2} k R_{0}^{2} \ln \left[1-\left(\frac{r_{i j}}{R_{0}}\right)^{2}\right], & r_{i j} \leq R_{0} \\
+\infty, & r_{i j} \geq R_{0},
\end{array}\right.
$$

where $k=30 \epsilon$ is the spring constant for the FENE bond, $R_{0}=1.5 \sigma$ is the limit of the bond extension, and $r_{i j}$ is the distance between particles $i$ and $j$. The LJ potential cutoff distance was $r_{c}=2.5 \sigma$. The minimum of the bond potential along the chain occurs at a position $l_{\min } \approx 0.97 \sigma$ which is different from the minimum position $r_{\min } \approx 1.13 \sigma$ of the LJ potential. The superposition of these two length scales prevents crystallization very efficiently, and the resulting structure of the melt and the corresponding glass resembles experimental data ${ }^{29,30}$ for polymer melts. 
A series of three different systems consisting of 10-, 20and 50-site molecules were studied. The system size was 108 or 864 molecules and the molecular temperature and reduced atomic site number density were $T=1.0$ and $\rho_{a}=0.84$, respectively. The state point was chosen to correspond to previous studies $^{27,31,32}$ of chain dynamics. These parameters remained constant through this series of simulations.

\section{B. Equations of motion}

The fifth-order Gear predictor corrector algorithm was employed to solve the equations of motion. ${ }^{33,34}$ The thermostatted equations of motion are given by ${ }^{24}$

$$
\dot{\mathbf{r}}_{i \alpha}=\frac{\mathbf{p}_{i \alpha}}{m_{i \alpha}}, \quad \dot{\mathbf{p}}_{i \alpha}=\mathbf{F}_{i \alpha}^{\mathrm{LJ}}+\mathbf{F}_{i \alpha}^{C / \mathrm{FENE}}-\zeta_{M} \frac{m_{i \alpha}}{M_{i}} \mathbf{p}_{i} .
$$

The force on a site is separated into three terms; $\mathbf{F}_{i \alpha}^{\mathrm{LJ}}$ is the contribution due to the Lennard-Jones type interactions on site $\alpha$ of molecule $i$ and $\mathbf{F}_{i \alpha}^{C / F E N E}$ is either the constraint force or the FENE bonding force. The details of the constraint algorithm for the FJC model have been discussed previously. ${ }^{35-37} \zeta_{M}$ is the molecular thermostat multiplier,

$$
\zeta_{M}=\frac{\sum_{i=1}^{N_{m}} \mathbf{F}_{i} \cdot \mathbf{p}_{i} / M_{i}}{\sum_{i=1}^{N_{M}} \mathbf{p}_{i}^{2} / M_{i}},
$$

which is derived from Gauss' principle of least constraint and acts to keep the molecular center of mass kinetic temperature $T_{M}$ constant. The molecular temperature $T_{M}$ is defined by

$$
T_{M}=\frac{1}{\left(d N_{m}-N_{c}\right) k_{B}} \sum_{i=1}^{N_{m}} \frac{\mathbf{p}_{i}^{2}}{m_{i}}
$$

where $d$ is the dimensionality of the system and $N_{c}=4$ is the number of constraints on the molecular center of mass degrees of freedom (i.e., constraints on the center of mass momenta and kinetic energy). We will not distinguish between molecular and atomic temperature, but simply use $T$ to indicate the temperature.

\section{Simulation details}

After an equilibration run of length $10^{6}$ time steps at low density, the molecular systems were compressed to the required density and re-equilibrated for $10^{6}$ time steps. A total of $1.4 \times 10^{7}$ time steps was used to compute the ACFs at each state point by ensemble averaging 14 independent runs, each of length $10^{6}$ steps. The transverse momentum density ACFs were computed over at least 20 reduced time units for 10 -site molecules and over at least 40 reduced time units for 50 -site molecules. The stress ACFs were computed over at least 40 and 80 reduced time units for 10-site and 50-site molecules, respectively. Several very long test runs over 200, 400 , and 600 reduced time units for 10-, 20-, and 50-site molecules were also carried out at very low temperatures where a much slower convergence of the integral stress ACF occurs. If the temperature of the polymer melt is too low, direct calculations of the complete stress ACF become unfeasible due to the enormously large relaxation times. Therefore, in addition to the ACFs, we have employed a widely used fitting procedure (see Sec. IV B) in which the stress $\mathrm{ACF}$ is extrapolated to much larger time values.

The transverse momentum density and stress ACFs were computed at wave-vectors $k_{y n}=2 \pi n / L_{y}$, where the mode number $n$ is from 0 to 40 with increment 2 and $L_{y}$ $=\left(N_{a} / \rho_{a}\right)^{1 / 3}$. For the remainder of this paper we drop the $n$ index in $k_{y n}$ for simplicity. The ACFs were Laplace transformed with respect to time using Filon's rule. ${ }^{38}$ Equation (2) was used to obtain the nonzero wave-vector viscosities and Eq. (8) was used to obtain the zero wave-vector viscosity. All the simulations in this work were carried out at zero frequency.

\section{RESULTS AND DISCUSSION}

The results presented below have been obtained by simulating 864 molecules in the isokinetic molecular dynamics ensemble. In order to validate our code and results, we reproduced and compared previous results for self-diffusion and wave-vector and frequency dependent viscosities where possible. Very good agreement was found in all cases. We must also note that all our correlation functions were evaluated for both 108 and 864 molecule systems in order to determine whether the results were system size dependent. No such evidence was observed within statistical uncertainties either at relatively high temperatures or down to the glass transition region.

\section{A. Self-diffusion and glass transition temperature estimation}

First, we note that a comparison of the velocity ACF of a 20-site FENE-LJ polymer melt with those of Hunt and Todd $^{39}$ at the lowest strain rate and for the highest temperature shows an excellent agreement. However, in order to limit the number of figures, we only show the results for the self-diffusion coefficient and omit displaying the velocity ACFs. The complete set of correlation function results are available from the authors and are presented in the Ph.D. thesis of Puscasu. ${ }^{40}$

The self-diffusion coefficient can be obtained either from the mean square displacement or via the Green-Kubo relation. In this work we evaluate the integral

$$
D=\frac{1}{3 m_{i}^{2}} \int_{0}^{\infty} d t\left\langle\mathbf{p}_{i}(t) \cdot \mathbf{p}_{i}(0)\right\rangle .
$$

The momenta $\mathbf{p}_{i}$ can be interpreted either as the momentum of an individual atom or as the center of mass momentum of a molecule. In this work we use the latter interpretation.

In Figs. 1(a)-1(c), the dependence of the self-diffusion coefficient as a function of temperature $T$ follows the expected behavior: higher temperatures lead to higher diffusion coefficients and $D$ decreases as $N_{s}$ increases. However, for the FJC-WCA system, the diffusion coefficient seems to vanish at a much lower temperature compared to the other systems, i.e., $T_{g}^{\mathrm{FJC}-\mathrm{WCA}}<0.01$ at $\rho=0.84$. Such systems exhibit a topologically induced glass transition which is controlled exclusively by chain structure. ${ }^{41-43}$ This explains why it appears the diffusion constant is approaching a limiting constant value at low temperature. ${ }^{44}$ For a hard core model the 

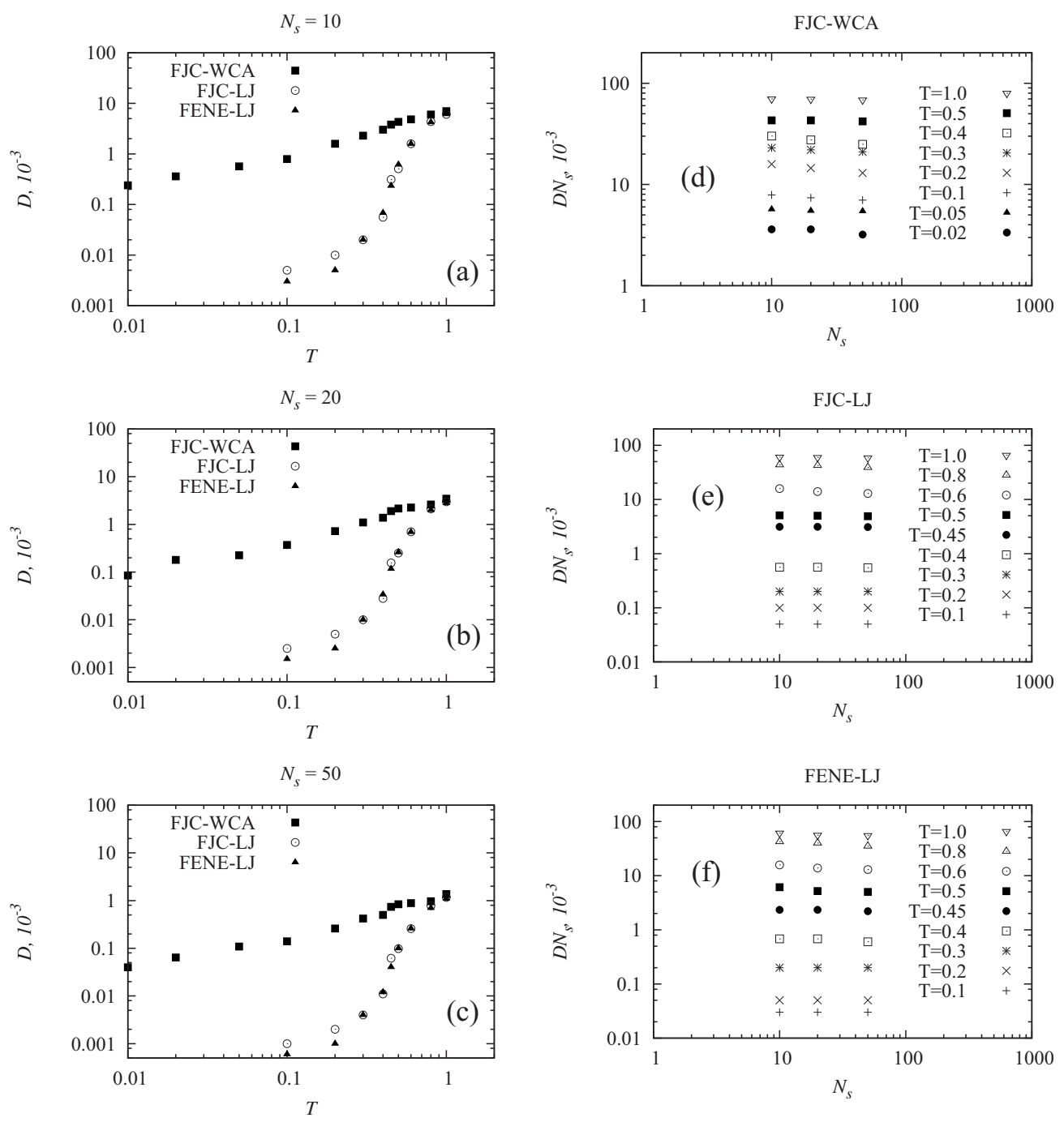

FIG. 1. Equilibrium diffusion coefficient $D$ for FJC-WCA, FJC-LJ, and FENE-LJ systems at different temperatures $T$ and for three chain lengths: (a) $D$ vs $T$ for $N_{s}=10$; (b) $D$ vs $T$ for $N_{s}=20$; (c) $D$ vs $T$ for $N_{s}=50$; (d) $D N_{s}$ vs $N_{s}$ for FJC-WCA; (e) $D N_{s}$ vs $N_{s}$ for FJC-LJ; (f) $D N_{s}$ vs $N_{s}$ for FENE-LJ. The dynamics of the systems are characteristic of the Rouse model.

temperatures and densities chosen here are too low to furnish sufficient packing constraints to induce the glass transition, hence the glass aspects are weak. Therefore, our results for the FJC-WCA model deal at best with a slightly supercooled liquid above the glass transition. The glass transition temperature for the FJC-LJ and FENE-LJ polymer melts is considerably higher. This is generally explained by the fact that the diffusion is significantly reduced by the second LJ coordination shell which falls within the attractive region of the potential. For these systems $T_{g}^{\mathrm{FJC} / \mathrm{FENE}-\mathrm{LJ}} \approx 0.40-0.45$ at $\rho$ $=0.84$. Later we find further evidence of the glass transition by investigating the zero wave-vector stress autocorrelation function. Our results for the self-diffusion are in agreement with those previously reported by Bennemann et al. ${ }^{45}$ for an identical system. It is also seen that for all chain lengths, Figs. 1(a)-1(c), the data points collapse onto one curve at around $T=1$, and above this temperature there is no difference in the diffusion for different model chains at the same $N_{s}$, as found previously by Kremer and Grest. ${ }^{27}$

The FJC-WCA system is not likely to undergo a sharp transition at the temperatures simulated here, therefore the glass transition temperature should only be considered as an estimate of the interval associated with the glass transition. These results are consistent with those of Bulacu et al. ${ }^{46}$ who suggested that $T_{g}$ for a similar system was 0.05 . To obtain a more accurate estimation of $T_{g}$, it would be necessary to either conduct simulations at lower temperatures or to fit $D$ as a function of $T$ with different theoretical or empirical equations. ${ }^{1}$ Both methods are somewhat troublesome; equilibrium MD simulations are not accurate enough to distinguish between exponentially small and strictly zero values of $D$ and the fitting results are weakly dependent on the temperature interval used. Since $T_{g}$ is not our primary interest we did not perform such estimations.

Figures 1(d)-1(f) show the self-diffusion coefficient as a function of chain length at temperatures ranging from well below $T_{g}$ to above $T_{g}$. At all temperatures, the chains behave approximately according to the Rouse theory for all three system types. The diffusion coefficient is inversely proportional to the chain length (hence viscosity should be directly proportional to $N_{s}$ ) which is consistent with our polymeric chains being not long enough to cover the crossover regime from the nonentangled to the entangled state, in agreement with previous observations. ${ }^{47}$ 

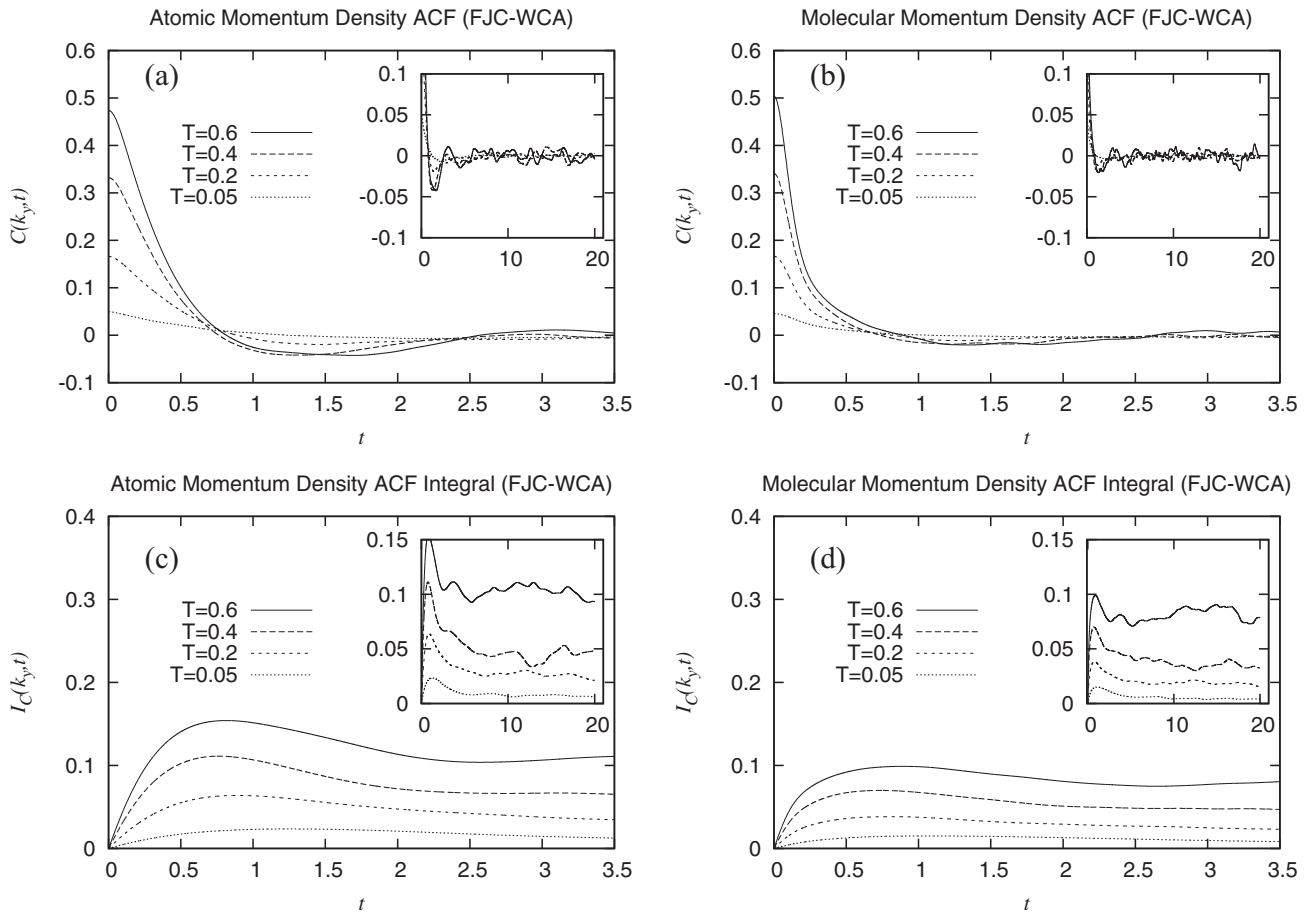

FIG. 2. Momentum density ACFs as a function of temperature for a 10-site FJC-WCA polymer melt: (a) atomic transverse momentum density ACFs; (b) molecular transverse momentum density ACFs; (c) integral of the atomic transverse momentum density ACFs; (d) integral of the molecular transverse momentum density ACFs. The mode number is $n=2$. The insets show the long-time behavior.

\section{B. Momentum density and stress correlation functions}

The transverse momentum density ACFs expressed by Eq. (3) and their running integrals, Eq. (4), are shown in Figs. 2-4 for the FJC-WCA, FJC-LJ, and FENE-LJ systems, respectively. Again, in order to limit the number of figures, we only show here results for a ten-site system and for mode number $n=2$. While $C_{\perp}\left(k_{y}, t\right)$ in all three models look similar, the running integrals in the case of FJC-WCA polymer chains behave differently from FJC-LJ and FENE-LJ chains as we lower the temperature. Figures 2(a)-2(d) show clear evidence that the FJC-WCA system, which contains only the repulsive part of the LJ potential, is unlikely to undergo a
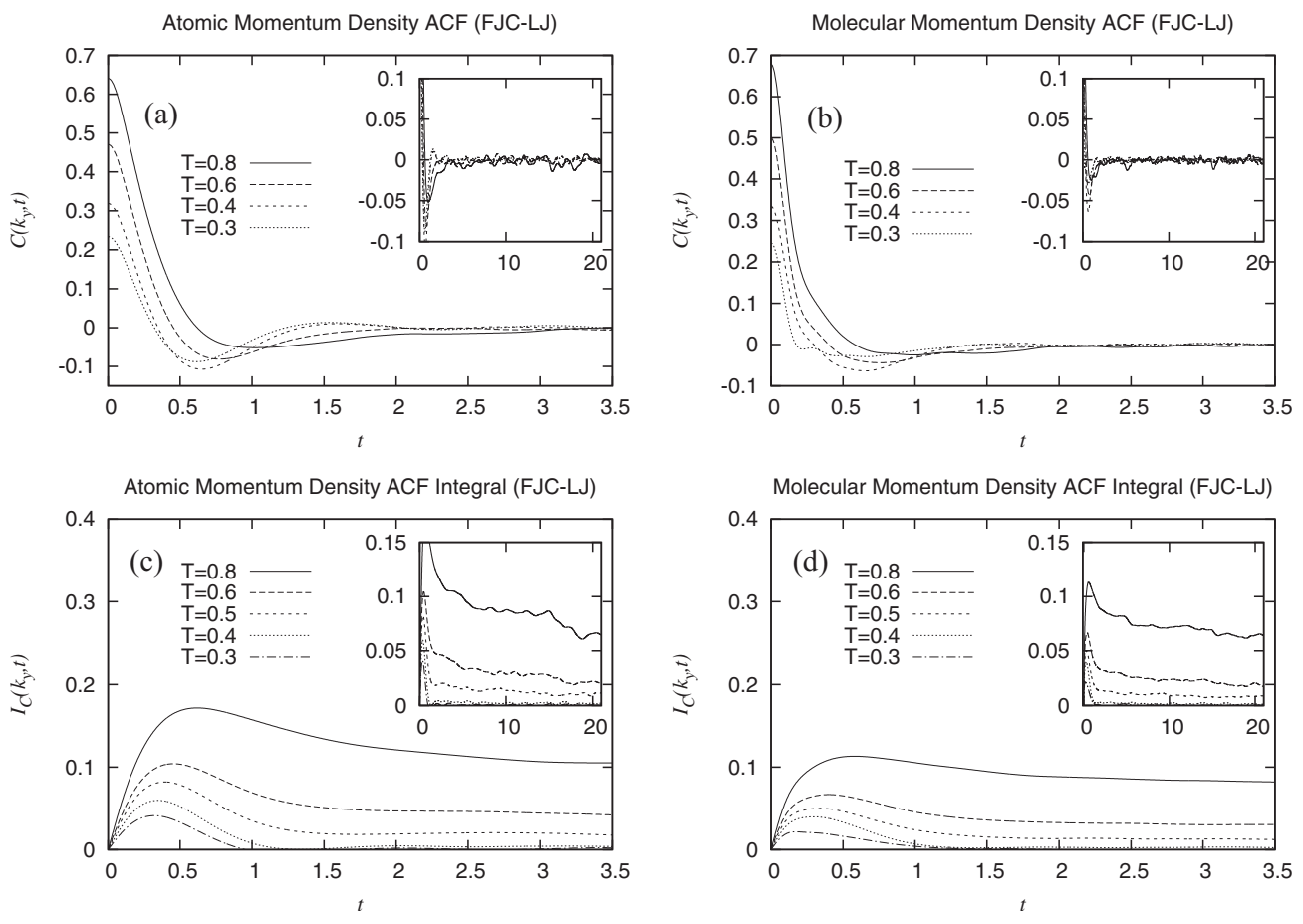

FIG. 3. Momentum density ACFs as a function of temperature for a 10-site FJC-LJ polymer melt: (a) atomic transverse momentum density ACFs; (b) molecular transverse momentum density ACFs; (c) integral of the atomic transverse momentum density ACFs; (d) integral of the molecular transverse momentum density ACFs. The mode number is $n=2$. The insets show the long-time behavior. 

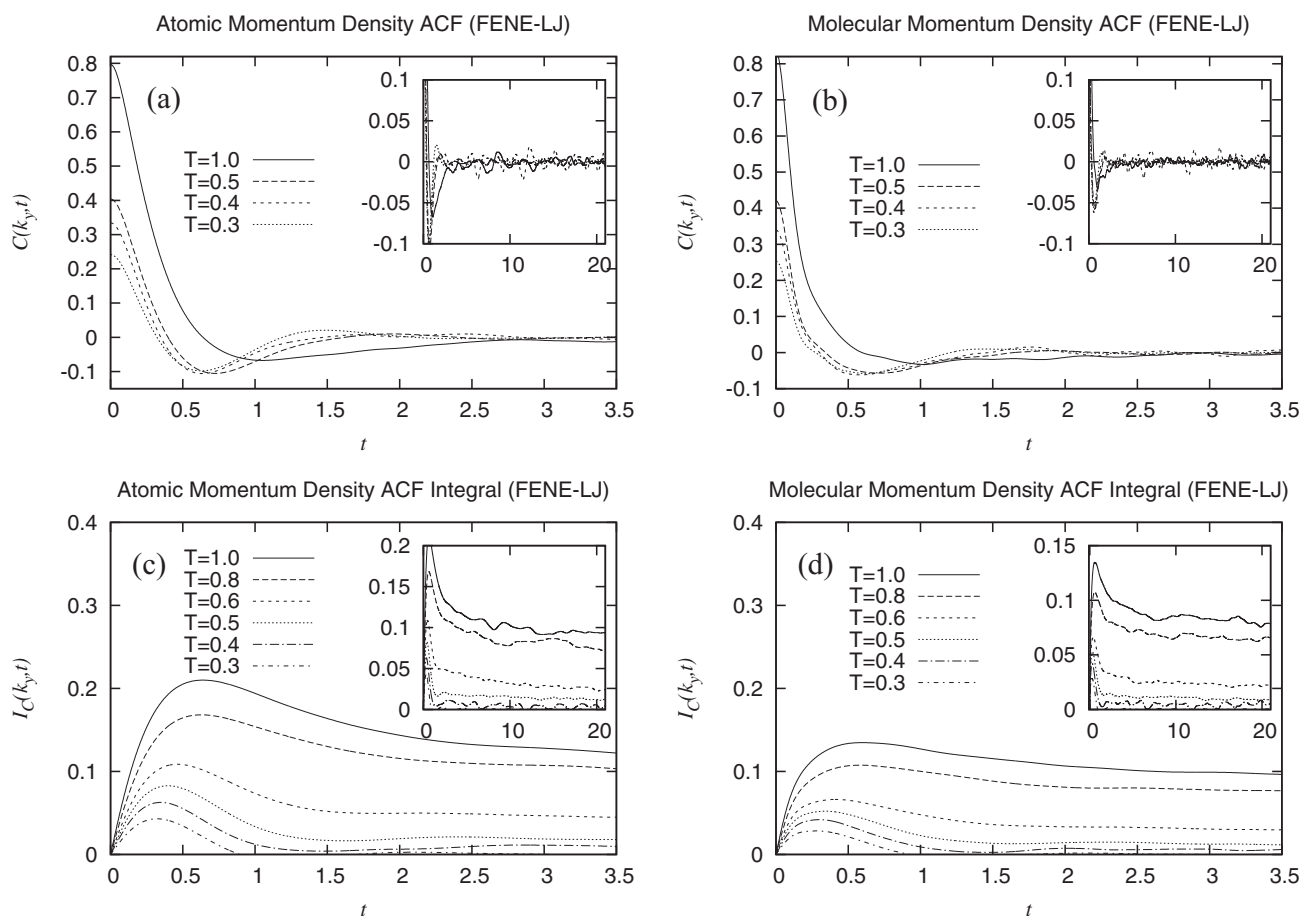

FIG. 4. Momentum density ACFs as a function of temperature for a 10-site FENE-LJ polymer melt: (a) atomic transverse momentum density ACFs; (b) molecular transverse momentum density ACFs; (c) integral of the atomic transverse momentum density ACFs; (d) integral of the molecular transverse momentum density ACFs. The mode number is $n=2$. The insets show the long-time behavior.

structural arrest even at $T=0.05$. A further examination of Figs. 2(a), 2(b), 3(a), 3(b), 4(a), and 4(b) shows that in the atomic representation the zero time values of the momentum density ACFs differ slightly from the molecular ones. This is a consequence of correlations between the momenta of different sites on the same molecule that are involved in bond angle or bond length constraints [see Evans and Morriss, Chap. 4, Eq. (37) in Ref. 10]. As a consequence, there is no longer a simple relationship between the temperature and the zero time value of the momentum density ACF. Therefore the $y$-intercepts in Figs. 2-4 are computed from the correlation function in Eq. (3) and not from the temperature, Eq. (7). ${ }^{18}$

The stress autocorrelation functions expressed by Eq. (9) and their running integrals expressed by Eq. (11) are shown in Figs. 5-7 for FJC-WCA, FJC-LJ, and FENE-LJ chains, respectively, at $k_{y}=0$. In contrast to the FJC-WCA model, in the FJC-LJ and FENE-LJ chains, the relaxation time increases monotonically with decreasing temperature until a specific temperature is reached. At this temperature a sudden increase of the relaxation time occurs indicating a strong slowing down of the chain dynamics. This temperature is very similar to the one obtained from the vanishing of the diffusion coefficient $D$, Figs. 1(a)-1(c). In addition to the above mentioned stress correlation functions calculated over 40 reduced time units, we also performed longer test runs over at least 200, 400, and 600 reduced time units for 10-, $20-$, and 50-site molecules, respectively. The log-log atomic stress ACFs, for three different chain lengths, are displayed in Figs. 8(a)-8(c) and their corresponding running integrals are displayed in Figs. 8(d)-8(f). As can be seen in Figs. 8(a)-8(c), the stress ACF exhibits a two step relaxation process as we lower the temperature, the so-called $\alpha$ and $\beta$ processes. The slowing down of relaxation and the development of the two step process occur for lower temperatures closer to the glass transition temperature. For the $\alpha$-relaxation time scale the mode coupling theory of the glass transition predicts a power law divergence. The critical behavior and the exponent of the $\alpha$ relaxation will be examined in Sec. IV C.

It is also necessary to extend the ACF in Figs. 8(d) $-8(\mathrm{f})$ to times longer than actually observed to ensure convergence of the integral. In such situations, the stress correlation functions are usually fitted to the Kohlarausch-Williams-Watts (or stretched-exponential) function above $T_{g}$ and to a power law form below $T_{g}{ }^{8}$ Instead of fitting the ACFs in Figs. $8(\mathrm{a})-8(\mathrm{c})$, we will use the running integrals, Figs. 8(d)-8(f), to tune the fitting parameters. The latter method reduces the noise and gives better convergence. The stretchedexponential functional form is

$$
I_{N}^{K W W}\left(k_{y}=0, t\right)=I_{N}(0,0)\left[1-\exp \left(-\gamma t^{\theta}\right)\right],
$$

and the power law functional form is

$$
I_{N}^{p o w}\left(k_{y}=0, t\right)=A+B t^{\phi} .
$$

It is important to note that the values of fitting parameters depend weakly on where the fit starts. The fits are shown in Fig. 8 and cover all times beyond $t=0.4$.

\section{Viscosity kernels in reciprocal space}

The reciprocal space kernels in the atomic formulation for FJC-LJ and FENE-LJ systems are shown in Fig. 9. The statistical reliability of the nonzero wave-vector kernel data increases as $k_{y}$ increases. The error bars for the nonzero wave-vector components are of the same size as the symbols 

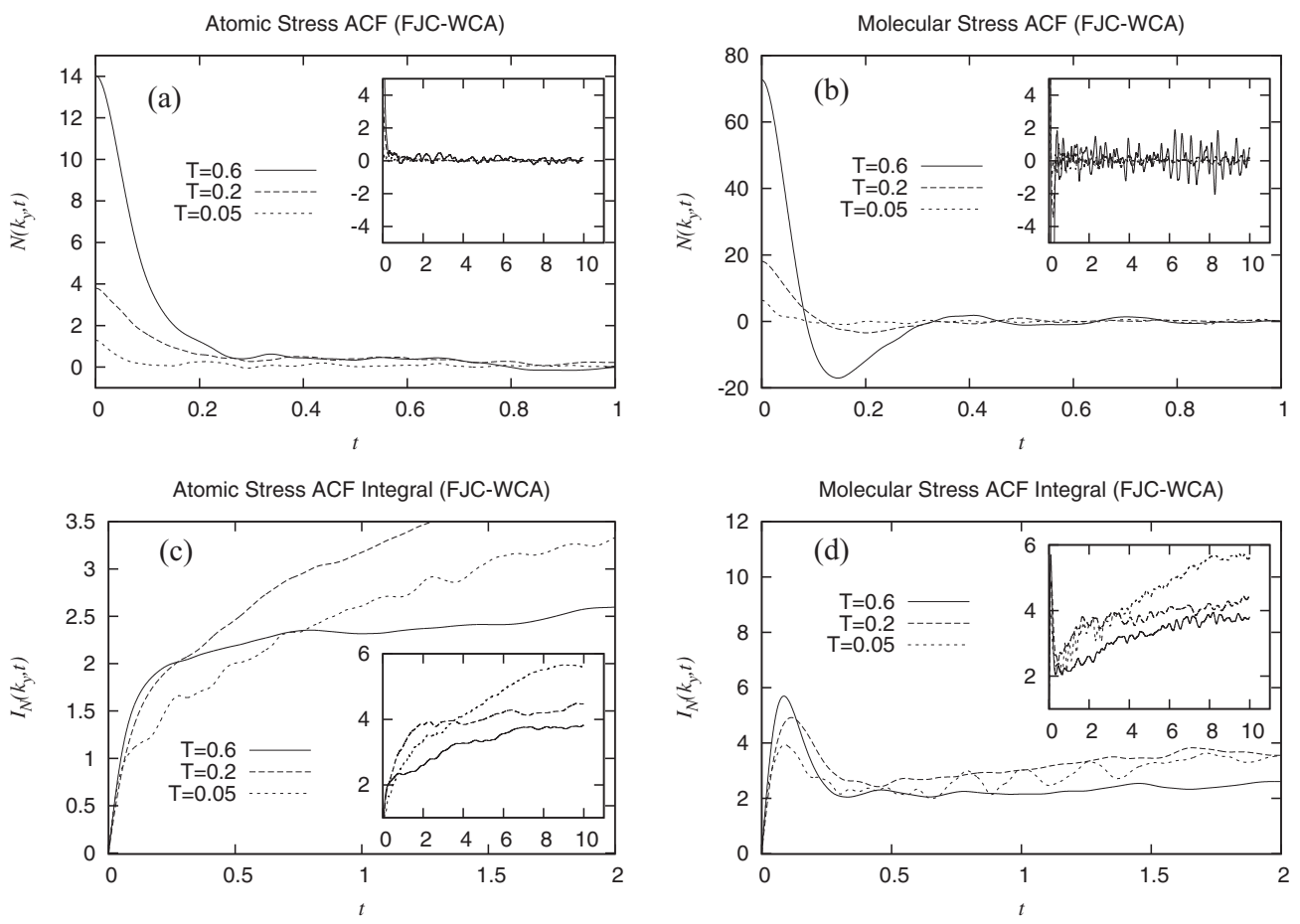

FIG. 5. Stress ACFs as a function of temperature for a 10-site FJC-WCA polymer melt at $k_{y}=0$ : (a) atomic stress ACFs; (b) molecular stress ACFs; (c) integral of the atomic stress ACFs; (d) integral of the molecular stress ACFs. The insets show the long-time behavior. The estimated glass transition temperature is $T_{g} \approx 0.40-0.45$.

and are therefore omitted in all figures. For the zero wavevector component the main source of error at temperatures approaching the glass transition is associated with the fitting procedure.

The kernels in Fig. 9 are normalized with respect to the zero wave-vector values which are given in Table I. For higher temperatures, our results for the zero wave-vector viscosity are in agreement with those reported previously by Daivis et $a .^{31}$ and Kröger et al., ${ }^{32}$ but are slightly lower compared to Wallace et al. ${ }^{8}$ especially for temperatures close to $T_{g}$. Some of the previously reported results are taken as limiting values of the shear rate dependent viscosities at zero
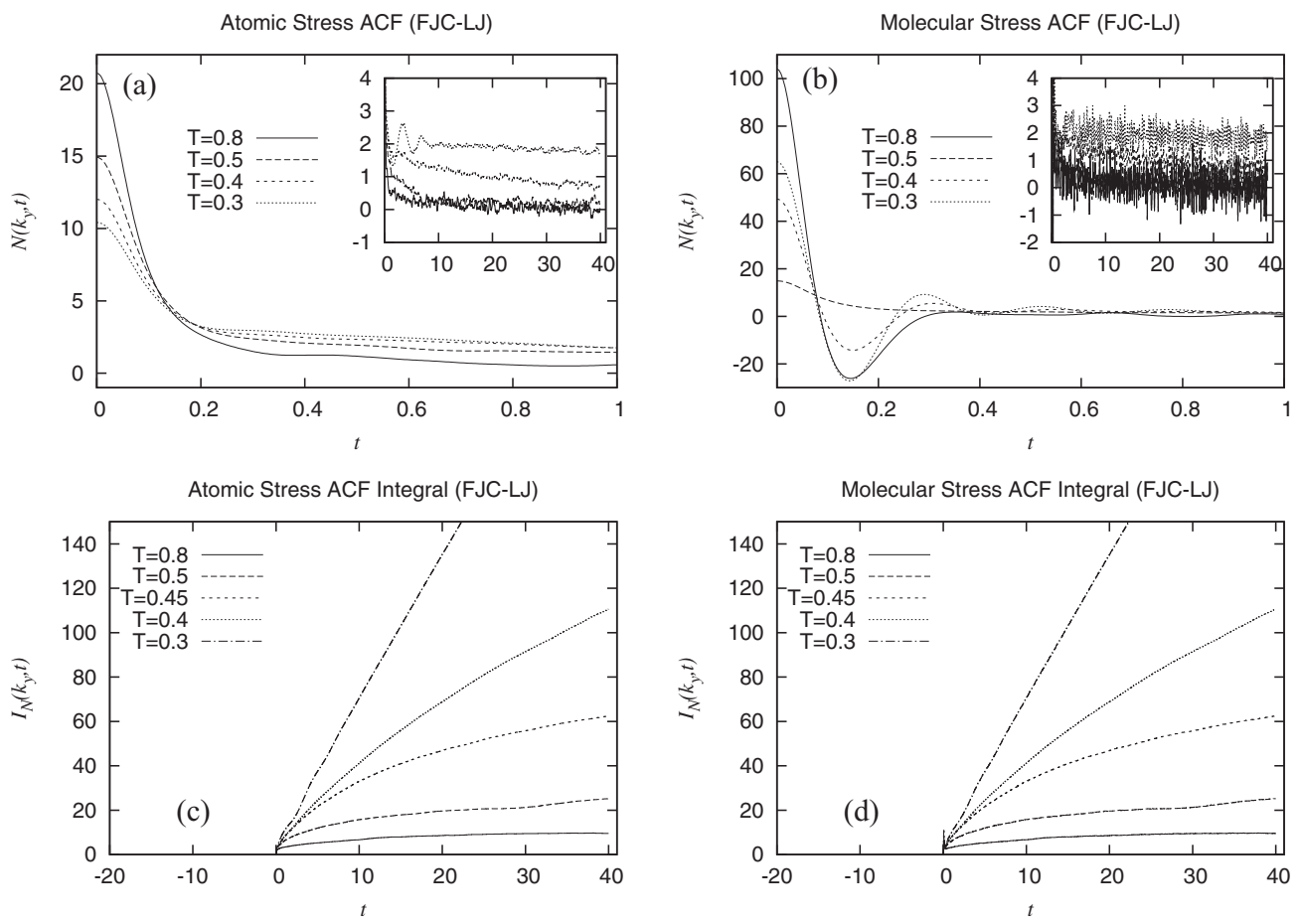

FIG. 6. Stress ACFs as a function of temperature for a 10-site FJC-LJ polymer melt at $k_{y}=0$ : (a) atomic stress ACFs; (b) Molecular stress ACFs; (c) integral of the atomic stress ACFs; (d) integral of the molecular stress ACFs. The insets show the long-time behaviors. Estimated glass transition temperature is $T_{g}$ $\approx 0.40-0.45$ 

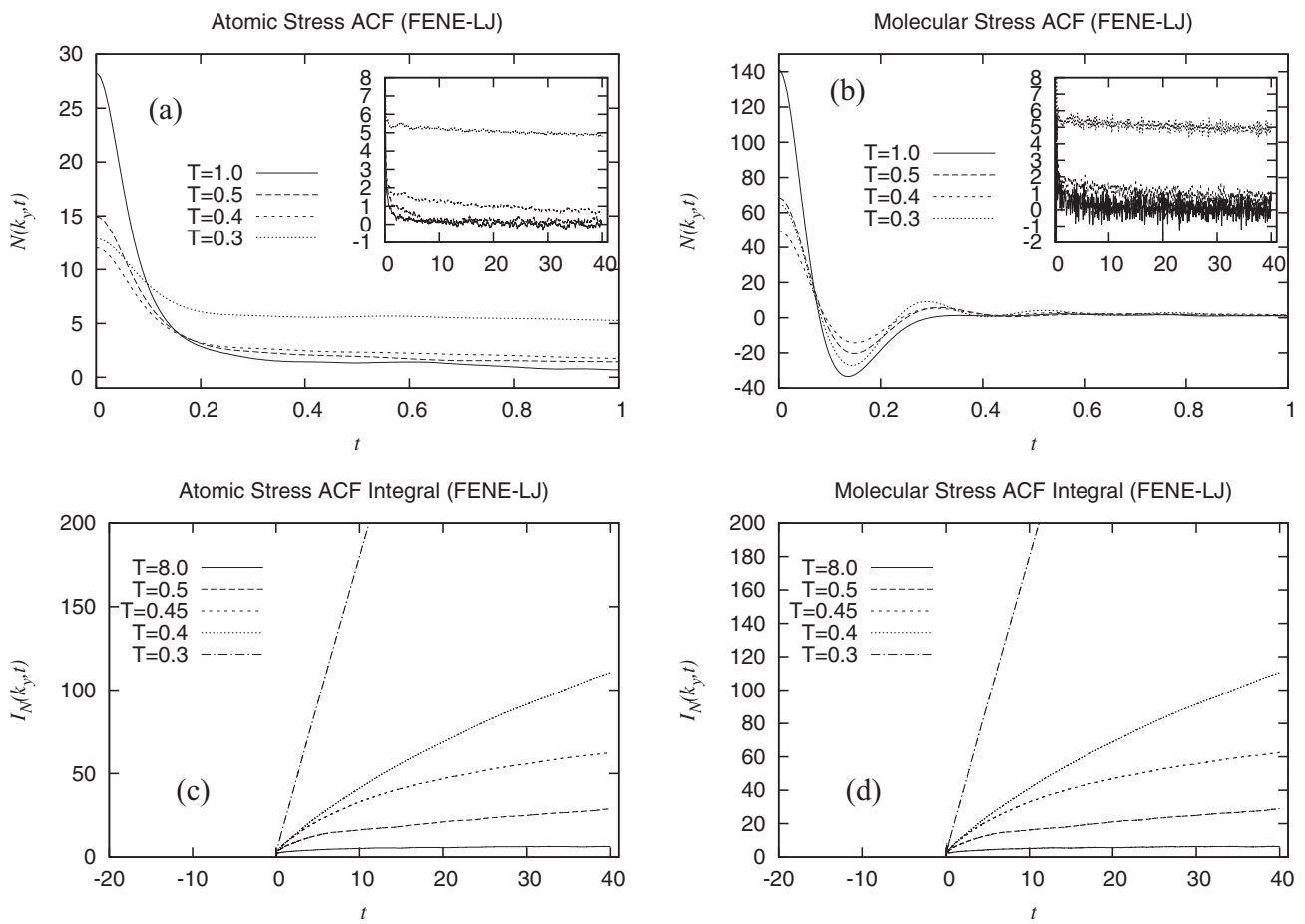

FIG. 7. Stress ACFs as a function of temperature for a 10-site FENE-LJ polymer melt at $k_{y}=0$ : (a) atomic stress ACFs; (b) molecular stress ACFs; (c) integral of the atomic stress ACFs; (d) integral of the molecular stress ACFs. The insets show the long-time behaviors. Estimated glass transition temperature is $T_{g}$ $\approx 0.40-0.45$.

strain rate. Nevertheless, they agree within the statistical uncertainty. The kernels of FJC-LJ and FENE-LJ chains are almost identical and therefore only FENE-LJ results are considered further in this paper. The similarity of these two models has also been confirmed by Hunt and Todd ${ }^{39,48}$ for shear viscosities, extensional viscosities, and the diffusion tensor.

We have previously found that two relatively simple functional forms could be used to fit the wave-vector dependent viscosity for a polymer melt, ${ }^{18}$ namely, a Gaussian function and a Lorentzian-type function. As pointed out in our previous work, ${ }^{18}$ the inclusion of additional terms in the Gaussian sum yields a better fit and makes the Gaussian function a suitable analytical approximation of the reciprocal space viscosity kernel. However, a Gaussian function with higher number of terms may result in unnatural behavior of the kernel with unphysical distortions in the real space kernels. By contrast, the Lorentzian-type function, expressed as

$$
\tilde{\eta}_{L}\left(k_{y}\right)=\frac{\eta_{0}}{1+\alpha\left|k_{y}\right|^{\beta}}, \quad \alpha, \beta \in \mathbb{R}_{+},
$$

shows a much smoother shape of the real space kernels and therefore is preferred in this paper. The results of the fitting are shown in Fig. 10 and Table II. In order to test the fitting procedure, the magnitude of the residuals was estimated using the residual standard deviation defined as $s_{r}$ $=\sqrt{\sum_{n=1}^{n_{s}} r^{2} /\left(n_{s}-n_{p}\right)}$, where $n_{s}$ is the number of data points, $n_{p}$ is the number of fitting parameters, and $r$ is the residual. ${ }^{49}$ After an iterative curve fitting procedure, the accurate estimation of $\eta_{0}$ was kept fixed allowing all other parameters in Eq. (22) to vary. In Fig. 11 we have plotted the fitting pa- rameters as a function of temperature for different chain lengths.

It is interesting to see whether $\eta_{0}$ obeys a VogelFulcher-Tamman (VFT)-law, ${ }^{5}$

$$
\eta^{V F T}(T)=\eta(\infty) \exp \left[c /\left(T-T_{0}\right)\right],
$$

where $\eta(\infty)$ is the shear viscosity at infinite temperature, $c$ is a constant, and $T_{0}$ is the temperature where the shear viscosity is expected to diverge. Fitting our data for all three chain lengths to Eq. (23), we obtained $\eta(\infty)=4.5 \pm 0.1, \quad c$ $=0.306 \pm 0.005$, and $T_{0}=0.393 \pm 0.001$ for a 10-site chain; $\eta(\infty)=18.2 \pm 4.8, c=0.16 \pm 0.04, T_{0}=0.395 \pm 0.007$ for a 20 site chain, and $\eta(\infty)=46.8 \pm 13.5, \quad c=0.14 \pm 0.05, \quad T_{0}$ $=0.40 \pm 0.01$ for a 50 -site chain. It should be mentioned that Varnik et al. ${ }^{5}$ reported $\eta(\infty)=13.23 \pm 0.13, c=0.615 \pm 0.036$, and $T_{0}=0.19 \pm 0.005$ for a similar ten-site chain model, however, they introduced an additional potential barrier acting on the monomers in a layered confined system. We can see that $T_{0} \approx 0.39$ is close to the estimated glass transition temperature from the calculations of self-diffusion. As shown in Fig. 11(a), the quality of the fit is very good for the ten-site molecule. However, as we have already mentioned, our data are not reliable below $T=0.45$. Here correlation functions decay very slowly in time and the fitting procedure must be extended over much larger delay times.

Table I clearly suggests that the Rouse model of the viscosity breaks down as we lower the temperature and it is therefore of interest to quantify the product $D \eta_{0}$ as a function of temperature and chain length. In order to quantify the Rouse failure and the break down of the Stokes-Einstein relation, we plot the product $D \eta_{0}$ as a function of $T$ and $N_{s}$ in Fig. 12. In contrast to the self-diffusion coefficient presented 

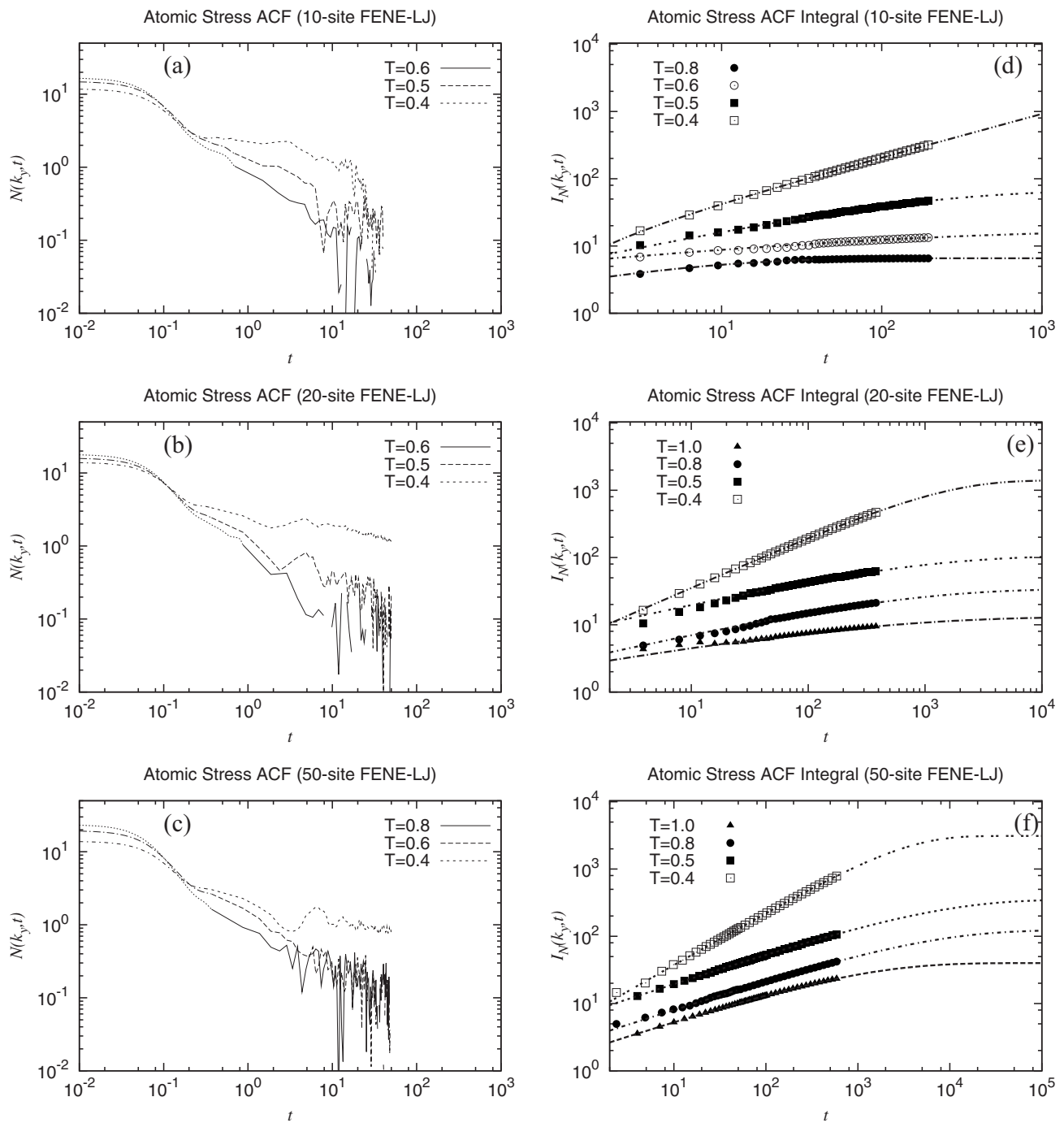

FIG. 8. Atomic stress ACFs and their running integrals at different temperatures for a 10-, 20-, and 50-site FENE-LJ polymer melt at $k_{y}=0$ : (a) 10-site stress ACFs; (b) 20-site stress ACFs; (c) 50-site stress ACFs; (d) 10-site integral stress ACFs; (e) 20-site integral stress ACFs; (f) 50-site integral stress ACFs. The time extensions in (d)-(f) have been obtained from a fit based on Eq. (20) except for $T=0.4$ in (a) where a power law, Eq. (21), was used. We note that in (e) and (f) the stretched-exponential fits the data slightly better compared to the power law, but due to the extremely large relaxation time, we suggest the former underestimates the limiting value. The value of the exponent characterizing the power law was found to be approximately $\theta=0.64$ for $N_{s}=10$.

in Sec. IV A for which the $N_{s}$ scaling of the center of mass $D$ is very simple and Rouse-like irrespective of temperature (Fig. 1), the Rouse-type scaling of the viscosity for FENE-LJ polymer melts is only seen at high temperatures with a deviation at $T=0.8$ (Table I). For the FJC-WCA systems the viscosity is Rouse-like down to around $T=0.4$. Below this temperature $D \eta_{0}$ follows a power law for both FENE-LJ and FJC-WCA systems. More evidence for the slowing down of the dynamics can be seen in the temperature dependence of $D \eta_{0}$ shown in Figs. 12(c) and 12(d). There are two reasons that can explain the observed behavior. One is related to the extrapolation of the stress ACF integrals. Recall that $\eta_{0}$ was taken as a limiting value based on stretched-exponential and power law fits, Eqs. (20) and (21), given in Figs. 8(d)-8(f), which become very sensitive to the interval used in the fitting in the absence of data at high time values as we lower the temperature below $T=0.6$. Hence the product $D \eta_{0}$ may not be accurate enough in the low- $T$ regime. When much longer simulations are employed, we expect a better agreement with the Rouse model prediction down to $T=0.5$. The second reason relates to the crossing over between the two distinct regimes. This occurs at a temperature $0.45<T$ $<0.5$ for FENE-LJ systems, Fig. 12(c), and $T<0.1$ for FJCWCA systems, Fig. 12(d), which is found to correspond well to the thermal decoupling associated with dynamic heterogeneity (as seen later in Sec. IV D). ${ }^{50}$

It is also of interest to extract an empirical mode coupling critical temperature for our simulation data. In general, for the $\alpha$-relaxation time scale the mode coupling theory (MCT) predicts a power law,

$$
D \propto\left(T-T_{c}\right)^{\varepsilon} .
$$

It should be mentioned that it is in principle possible to fit the self-diffusion data with the VFT expression similar to Eq. (23). In order to quantify the mode coupling critical temperature, we present the fits to the power law in Figs. 13(a) and 13(b) for the FJC-LJ and FENE-LJ systems, respectively. We found the power law exponent to be approximately $\varepsilon=1.5$ which is slightly lower compared to the values of 1.95 ( NVT ensemble) and 2.3 (NPT ensemble) obtained by Bennemann 

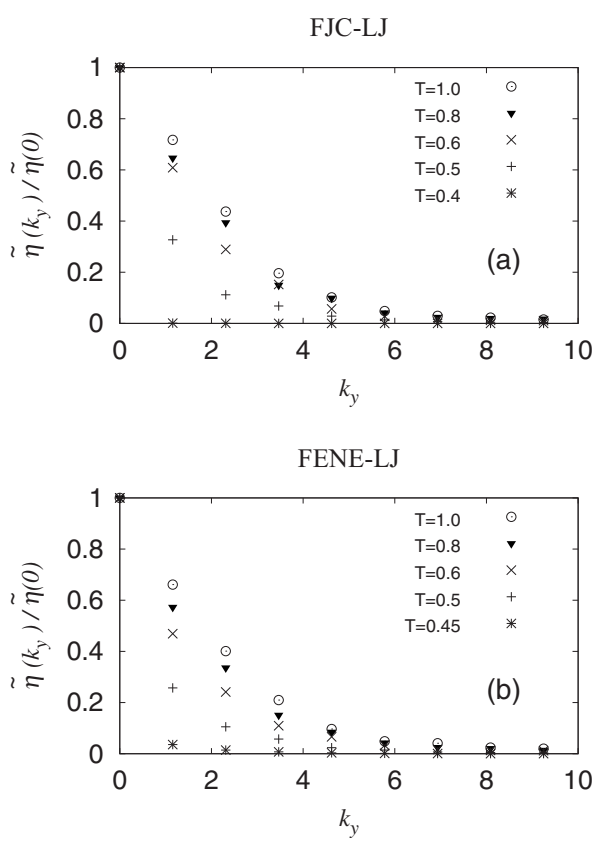

FIG. 9. Reciprocal space kernel of 10-site FJC-LJ and FENE-LJ melts at different temperatures in the atomic formalism: (a) normalized kernels for FJC-LJ; (b) normalized kernels for FENE-LJ.

et al. for a similar system. However, the mode coupling critical temperature is almost identical for $N_{s}=10$ despite the fact that our $T_{c}=0.39$ is slightly higher compared to the results of Bennemann et al., i.e., $T_{c}=0.32$ in the $N V T$ ensemble. A significantly lower mode coupling critical temperature is again in accord with what an extended mode coupling analysis would predict taking into account that MCT fails for deeply supercooled dynamics when activated barrier hopping dominates.

\section{Viscosity kernels in real space}

Since the viscosity kernel in reciprocal space is symmetric about the origin, it can be inverse transformed using the Fourier cosine transform, $F_{c}^{-1}[\ldots]$. The Fourier transform

TABLE I. Zero wave-vector, zero frequency viscosity obtained via Eq. (11) for different temperatures and chain lengths.

\begin{tabular}{|c|c|c|c|c|}
\hline \multirow[b]{2}{*}{ Number of sites } & \multirow{2}{*}{$\begin{array}{c}N_{s} \\
T\end{array}$} & 10 & 20 & 50 \\
\hline & & \multicolumn{3}{|c|}{$\eta_{0}$} \\
\hline \multirow[t]{6}{*}{ FENE-LJ } & 1.0 & 8.1 & 15.3 & 42.8 \\
\hline & 0.8 & 9.3 & 30.2 & 105 \\
\hline & 0.6 & 20 & 50 & 150 \\
\hline & 0.5 & 80 & 110 & 200 \\
\hline & 0.45 & & $\approx 10^{3}$ & \\
\hline & 0.4 & & $\approx 10^{4}$ & \\
\hline \multirow[t]{8}{*}{ FJC-WCA } & 1.0 & 8.09 & 15.2 & 41.2 \\
\hline & 0.8 & 9.3 & 17.0 & 44.5 \\
\hline & 0.6 & 10 & 18 & 47 \\
\hline & 0.4 & 12 & 21 & 52 \\
\hline & 0.2 & 17 & 29 & 61 \\
\hline & 0.1 & 25 & 38 & 72 \\
\hline & 0.05 & 41 & 56 & 93 \\
\hline & 0.02 & 67 & 81 & 117 \\
\hline
\end{tabular}

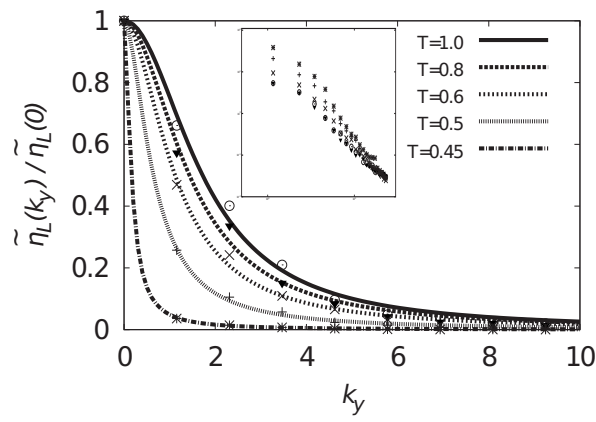

FIG. 10. The normalized reciprocal space kernel data of a 10-site FENE-LJ polymer melt and the fits to a Lorentzian-type functional form Eq. (22). Inset: The dependence of $\eta\left(k_{y}\right)$ as a function of temperature on a log-log scale.

preserves the even properties of the function which means that the viscosity kernel in physical space is also an even function. Since the integral is computed over an interval symmetric about the origin (i.e., $-\infty$ to $+\infty$ ), the transform can be expressed as

$$
F_{c}^{-1}\left[\widetilde{\eta}\left(k_{y}\right)\right]=\eta(y)=\sqrt{\frac{2}{\pi}} \int_{0}^{\infty} \widetilde{\eta}\left(k_{y}\right) \cos \left(k_{y} y\right) d k_{y} .
$$

While the inverse Fourier cosine transform of the Gaussian function exists ${ }^{51}$ and can be obtained analytically, ${ }^{18}$ the integral in Eq. (25) can only be evaluated numerically for the Lorentzian-type function, e.g., by a Simpson method. ${ }^{13,14}$

The real space kernels of a ten-site FENE-LJ chain are presented in Fig. 14. We can see that the width of the kernel is roughly 4-6 atomic diameters at $T=1.0$. As mentioned previously, the width of the reciprocal space kernel of both FJC-WCA and FENE-LJ polymer melts decreases with decreasing temperature. This means that the width of the real space kernel will increase with decreasing temperature. By cooling the melts down to $T=0.5$, the width of the kernel increases to at least 10 atomic diameters and then increases dramatically in the glass transition region. As stated previously, the normalized real space kernels for the FJC-WCA system are very close to each other upon decreasing the temperatures, and, since we are not confident that such a system will actually exhibit a glass transition within the temperature range considered here, we do not display them in this paper.

We have shown that the form of the fitting function used for the wave-vector dependent viscosity has a strong effect on the real space kernel's shape. ${ }^{18}$ As the temperature decreases, the choice of fitting function has a greater effect on the shape compared to the form of potential and consequently on the width of the kernel. For instance, kernels obtained from a two-term equal-weighted Gaussian functional form are slightly distorted in physical space and yield

TABLE II. Parameter values of the Lorentzian-type fit, Eq. (22), for a 10site FENE-LJ chain at different temperatures.

\begin{tabular}{lcccccc}
\hline \hline$T$ & 1.0 & 0.8 & 0.6 & 0.5 & 0.45 & 0.4 \\
\hline$\eta_{0}$ & 8.1 & 9.3 & 20 & 80 & $10^{3}$ & $10^{4}$ \\
$\alpha$ & 0.327 & 0.497 & 0.811 & 2.182 & 19.5 & 55.5 \\
$\beta$ & 2.057 & 1.940 & 1.854 & 1.777 & 1.639 & 1.200 \\
\hline \hline
\end{tabular}



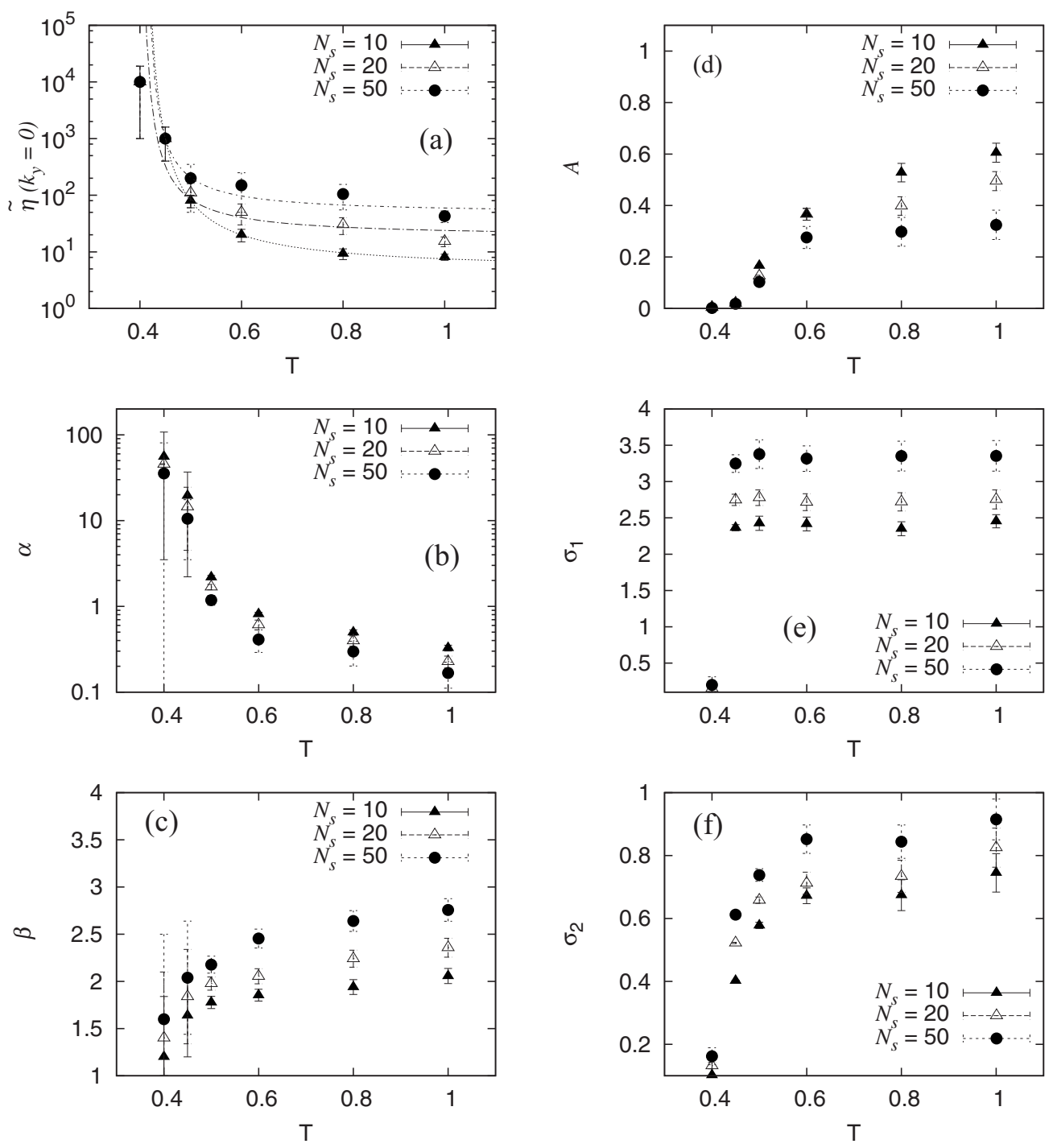

FIG. 11. Temperature dependence of the fitting parameters as a function of chain length for a FENE-LJ polymer melt in the atomic formalism: (a) temperature dependence of zero wave-vector, zero frequency viscosity. Also shown are best fits with the VFT expression Eq. (23); [(b) and (c)] temperature dependence of parameters $\alpha$ and $\beta$ Eq. (22); [(d)-(f)] temperature dependence of parameters $A, \sigma_{1}$, and $\sigma_{2}$ (Ref. 18), respectively. In (a) the error bars are based on averages over the test runs and in (b)-(f) the errors represent the asymptotic standard errors.

smaller widths. This problem could be easily solved by including more terms in the Gaussian form. However, this introduces undesired additional fitting parameters with insignificant improvement in the overall shape at temperatures above $T=0.5$.

For the FENE-LJ chain, the trends with chain length and the dependence of the results upon the choice of functional form for the real space are similar to those at state point $\rho$ $=0.84, T=1.0$ presented in Ref. 18. The shape of the kernels varies slightly with the number of sites per chain for the range of chain lengths considered here. Although the Gaussian and Lorentzian-type functional forms predict different shapes of the real space kernel, in general, and the $\eta(y=0)$ value, in particular, the local effective viscosities, $\eta_{0}$ $=\int_{-\infty}^{\infty} \eta(y) d y$, shown in Table III were in good agreement with the simulated values given in Table I for the above mentioned state point and for all molecular weights. However, we stress that care must be taken when the Lorentziantype function is inverse Fourier transformed over a very narrow function as it is very sensitive to the interval, number of points, and truncation used in integration.
Additional insight into the relationship between the viscosity kernel and the structure of the fluid can be gained by considering a scaling factor that accounts for the structural properties. Structural properties that we have investigated include the radial distribution functions (RDFs),

$$
g(r)=\left\langle\frac{\sum_{i=1}^{N} \sum_{j>1}^{N} \delta\left(\left|\mathbf{r}-\mathbf{r}_{i j}\right|\right)}{4 \pi r^{2} N \rho}\right\rangle .
$$

For an atomic description $\mathbf{r}_{i j}$ is the distance vector between atoms $i$ and $j, N$ is the total number of atoms, and $\rho$ is the atomic number density. For a molecular fluid $\mathbf{r}_{i j}$ is the distance vector between the centers of mass of molecules $i$ and $j, N$ is the total number of molecules, and $\rho$ is the molecular density.

We define a spatial correlation length $\xi_{g}$, which is used to normalize the distance in real space in order to compare the viscosity kernels for different structures. We define the structural scaling factor by ${ }^{14}$ 
FENE-LJ

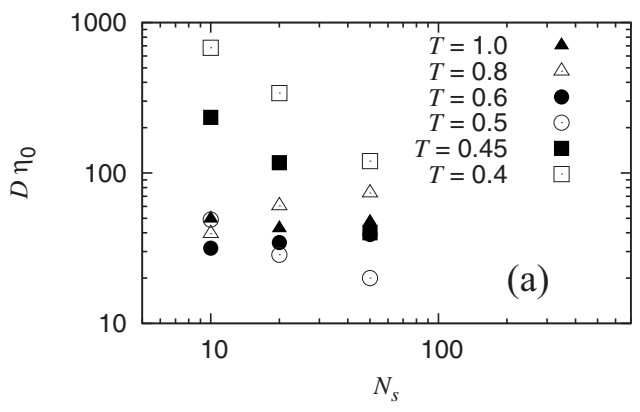

FENE-LJ

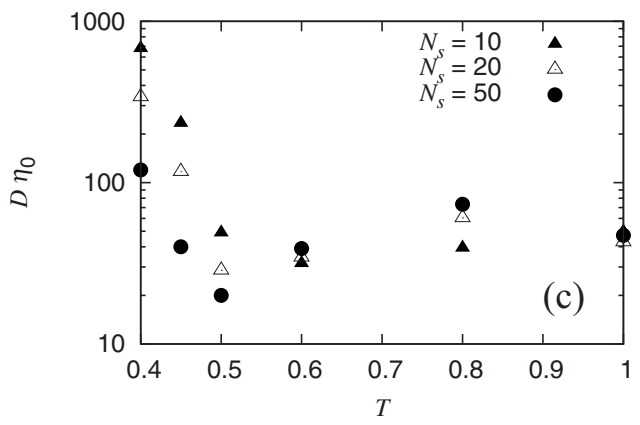

FJC-WCA

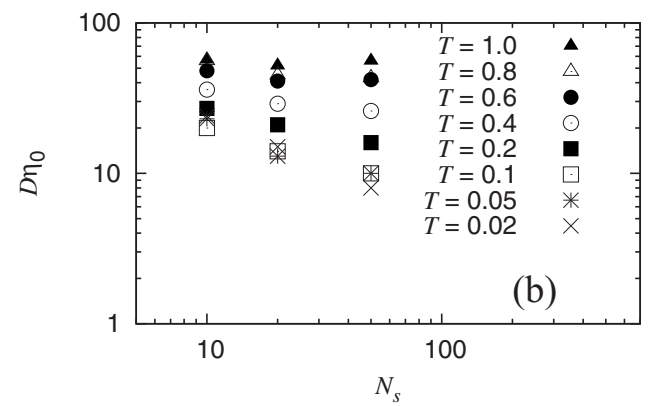

FJC-WCA

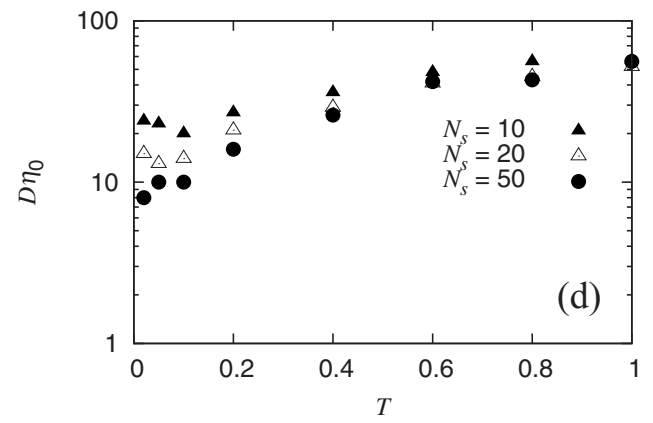

FIG. 12. Temperature and chain length dependence of $D \eta_{0}$ : (a) $D \eta_{0}$ vs $N$ for FENE-LJ polymer melts; (b) $D \eta_{0}$ vs $N$ for FENE-WCA polymer melts; (c) $D \eta_{0}$ vs $T$ for FENE-LJ polymer melts; (d) $D \eta_{0}$ vs $T$ for FENE-WCA polymer melts.

$$
\xi_{g}=\frac{\int_{0}^{\infty} r[g(r)-1]^{2} d r}{\int_{0}^{\infty}[g(r)-1]^{2} d r}
$$

which is a measure of the range over which the correlation function decays to 1 and therefore could be regarded as a correlation length of the radial distribution function.

Our radial distribution functions presented in Fig. 15 are in good agreement with those published previously for a similar polymer system. ${ }^{45,46}$ Berthier and Tarjus, ${ }^{52}$ by con-
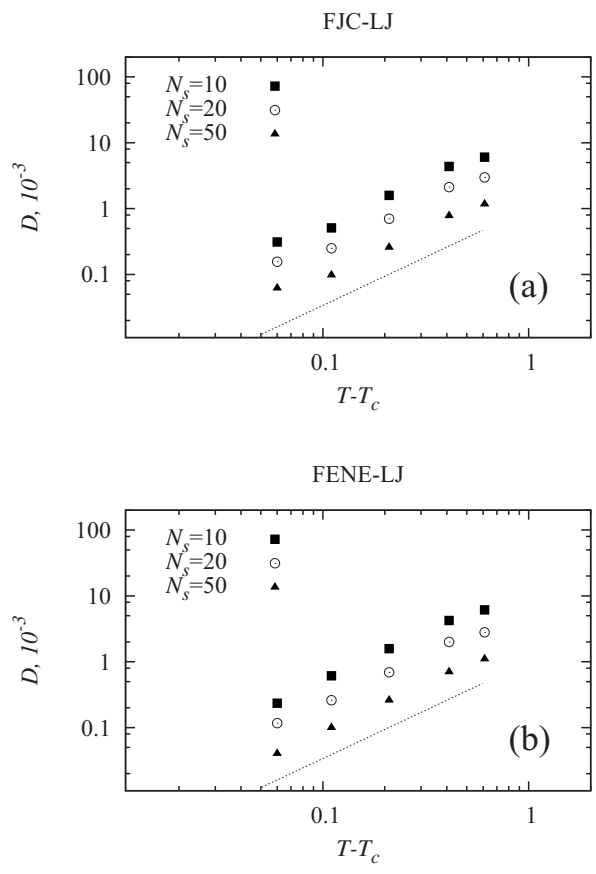

FIG. 13. Critical behavior of the self-diffusion coefficient for (a) FJC-LJ and (b) FENE-LJ polymer melts. $T_{c}=0.39$. Slope $\varepsilon=1.5$. trast, do not see significant changes of the radial distribution function with cooling. However, Berthier and Tarjus have looked at an atomic LJ mixture and therefore we cannot make direct comparisons. Nevertheless, we do believe that the structure of the polymer systems we have investigated does not change significantly with $T$. The radial distribution functions shown in Fig. 15 only indicates the onset of a supercooled state (the LJ peaks become more evident) without signs of crystallization (no intermediary peaks). The RDFs for $N_{s}=50$ in Fig. 15 show sharp peaks due to bonds $(l=1.0 \sigma)$ and LJ shells. The second LJ coordination shell is visible in a peak at $r \approx 2 \sigma . \xi_{g}$ increases as we lower the temperature, from 0.582 at $T=1.0$ to 0.836 at $T=0.4$ for a ten-site molecule and only slightly increases as we enlarge the polymers from 10 to 50 sites per molecule. The static scaling factor based on the pair distribution function slightly decreases with temperature simply because of the peaks in $g(r)$ which attenuate as we raise the temperature. It is not a

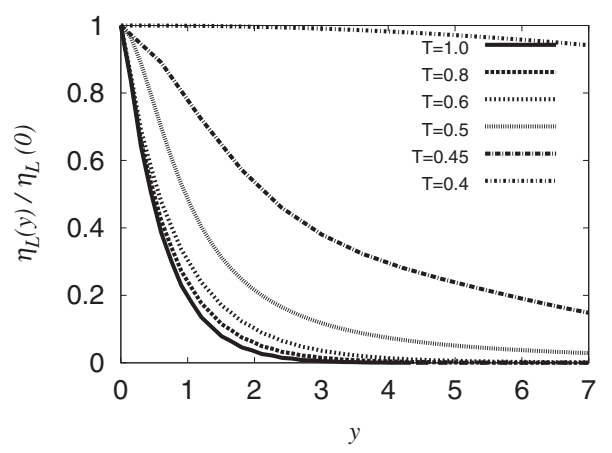

FIG. 14. Real space kernel of a 10-site FENE-LJ polymer melt obtained numerically from the Lorentzian-type functional form, Eq. (22), for different temperatures and normalized by $\eta(y=0)$. 
TABLE III. Zero shear rate viscosities $\eta_{0}$ evaluated numerically from a Lorentzian-type function, Eq. (22).

\begin{tabular}{|c|c|c|c|c|c|c|c|c|}
\hline$T$ & 1.0 & 0.8 & 0.6 & 0.5 & 0.45 & 0.4 & 0.2 & 0.02 \\
\hline & \multicolumn{8}{|c|}{ FENE-LJ } \\
\hline$N_{s}=10$ & 8.1 & 9.2 & 28 & 70 & 700 & $0.8 \times 10^{4}$ & $\ldots$ & $\cdots$ \\
\hline$N_{s}=20$ & 15.5 & 31.0 & 55 & 119 & 900 & $1.0 \times 10^{4}$ & $\cdots$ & $\cdots$ \\
\hline \multirow{2}{*}{$N_{s}=50$} & 42.8 & 105 & 150 & 223 & $1.2 \times 10^{3}$ & $1.3 \times 10^{4}$ & $\cdots$ & $\cdots$ \\
\hline & \multicolumn{8}{|c|}{ FJC-WCA } \\
\hline$N_{s}=10$ & 8.1 & 9.2 & 12 & $\cdots$ & $\cdots$ & 11 & 18 & 69 \\
\hline$N_{s}=20$ & 15.9 & 17.2 & 22 & $\ldots$ & $\cdots$ & 21 & 30 & 84 \\
\hline$N_{s}=50$ & 42.4 & 45.8 & 50 & $\cdots$ & $\cdots$ & 53 & 63 & 131 \\
\hline
\end{tabular}

complete "structural" representation and must be therefore treated as an attempt to introduce structural scaling. In addition, we found in our previous work ${ }^{18}$ that the intermolecular contribution in $g(r)$ leads to a completely different picture of the static scaling factor. Consequently, a deeper analysis of the structural scaling for different complex fluids is needed.

The real space kernels shown in Figs. 14 are normalized with respect to $\xi_{g}$ and replotted in Fig. 17(a). We can see that despite the fact that the structural scaling procedure has slightly reduced the difference between the real space kernels in the high temperature region $0.6 \leq T \leq 1.0$ and preserved their features, it does not completely remove this difference. This suggests that the width of the kernel does not only depend on the site-site correlations. The static scaling factor based on the pair distribution function, which is generally related to liquid structure, must be extended to include correlations such as alignment and orientation correlations. Furthermore, as the temperature approaches the glass transition region $T<0.5$, the structural scaling must be further generalized in order to suggest a universal kernel function.

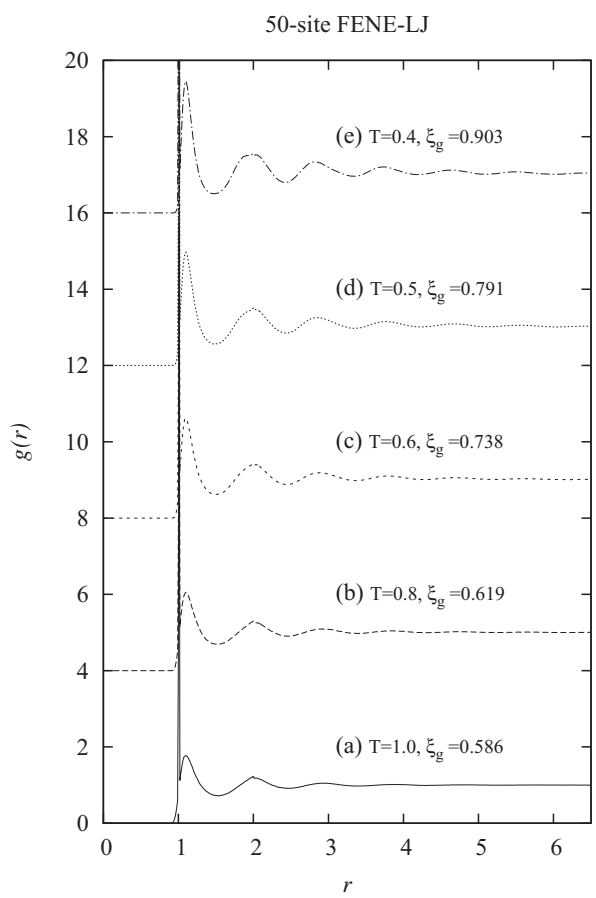

FIG. 15. Total intra- and intermolecular radial distribution function $g(r)$ and scaling factors $\xi_{g}$, as functions of temperature for a 50-site FENE-LJ polymer melt. For clarity, the RDFs are shifted upwards by four units in (b)-(e). A complete set of scaling factors are plotted in Fig. 16.
Such an approach could be based on the idea of dynamic heterogeneity which states that the dynamics of a glass former is governed by dynamic spatial correlations in contrast to the assumption of homogeneity of mode coupling theories. ${ }^{53}$ Therefore, a dynamic scaling factor must be employed. Such a scaling factor can be extracted for instance from Eq. (22) by nondimensionalizing $k_{y}$ by a length we will call $\zeta$,

$$
\zeta=\alpha^{1 / \beta} .
$$

The temperature dependence of $\zeta$ for different chain lengths is given in Fig. 16(b). We can see an exponential increase in the length scales as we approach the glass transition region. This confirms the existence of a dynamic heterogeneity in the system which implies that the increase in time scales as the glass transition is approached is associated with growing length scales of dynamically, not statically, correlated regions of space. ${ }^{54}$ Surprisingly, the temperature dependence of the dynamic scaling factor $\zeta$ looks very similar to the temperature dependence of the coherence length $\ell{ }^{50}$ The coherence length $\ell$ was associated with the ordering of the liquid's dynamics by measuring spatial correlations between indi-
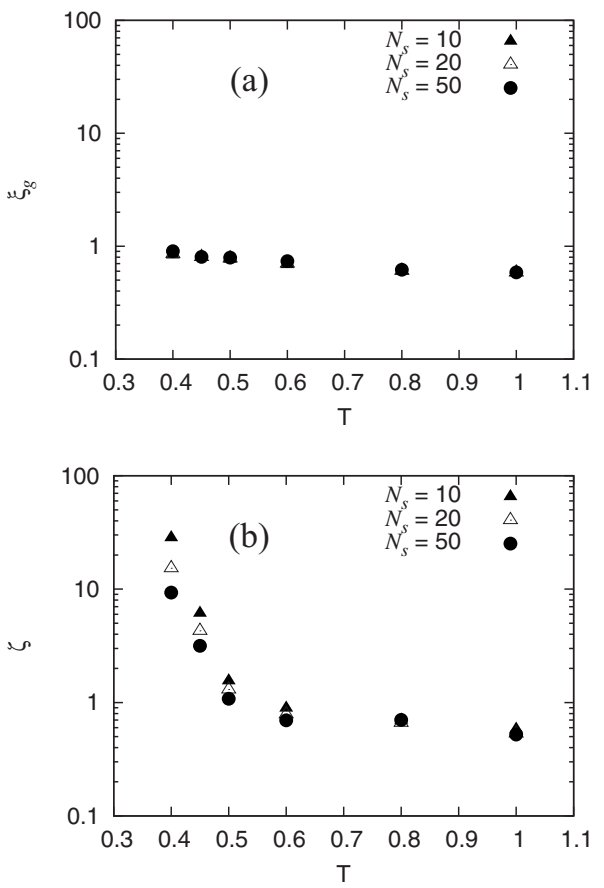

FIG. 16. Temperature dependence of the (a) static scaling factor $\xi_{g}$, Eq. (27), and (b) dynamic scaling factor $\zeta$, Eq. (28), for different chain lengths. 

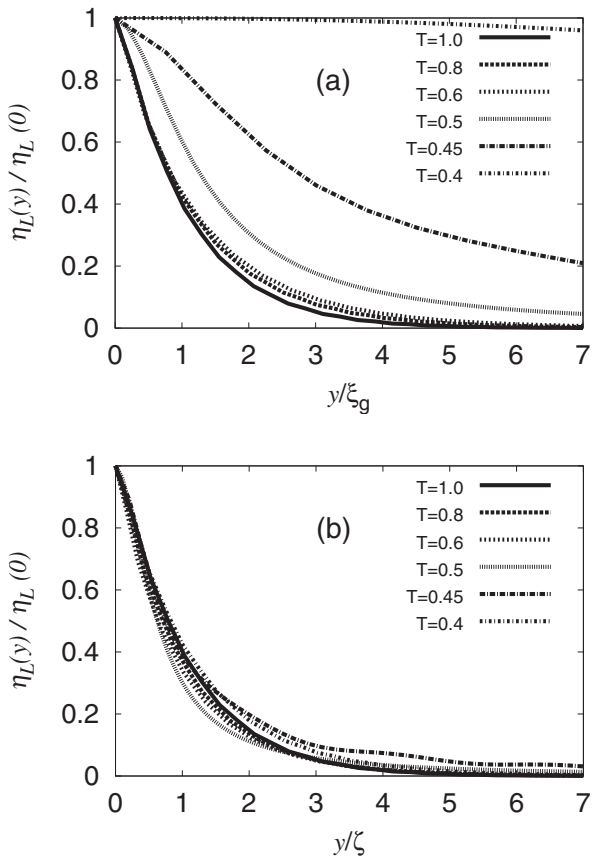

FIG. 17. Real space kernel of a 10-site FENE-LJ melt obtained numerically from the Lorentzian-type functional form, Eq. (22), at different temperatures and normalized by the (a) static factor $\xi_{g}$, Eq. (27) and (b) dynamic factor $\zeta$, Eq. (28).

vidual particle relaxations ${ }^{53}$ and calculated from the wavevector dependence of a correlator based on the standard self-intermediate scattering function. ${ }^{50,55}$

A further confirmation of the collective or cooperative dynamics can be seen in Fig. 17(b). The real space viscosity kernels fall essentially onto one curve for the entire range of temperatures. From Fig. 17, we can draw the conclusion that the behavior of the relaxation time changes from the high temperature to low temperature behavior close to the onset of a critical point responsible for the existence of the glass state. Thus, the viscosity of a supercooled liquid increases rapidly as temperature is lowered because the dynamics becomes increasingly spatially correlated (or nonlocal). The frequency dependence of the viscosity also undergoes a strong change as a liquid is supercooled and eventually solidified. ${ }^{56}$ The broadening of the real space viscosity kernel is accompanied by the emergence of a very slowly decaying tail in the stress $\mathrm{ACF}$, which eventually becomes a constant offset.

\section{CONCLUSION}

This work has been devoted to the analysis of the nonlocal viscosity kernel of polymeric fluids, when cooled toward their glass transition temperature using equilibrium molecular dynamics simulation. This study confirms the previous results for the self-diffusion coefficient and glass transition temperature and points out the importance of including the attractive part in the potential ${ }^{52}$ in order to achieve a glassy state in which to study the shape of the kernels.

The values obtained for the self-diffusion coefficients and the glass transition temperatures are in good agreement with those available in the literature. For the FJC-WCA sys- tem, we observe no glasslike enhancement except at extremely low temperatures of less than 0.01 . This value is not evident from the stress ACF.

The evaluation of the zero wave-vector viscosity involves calculations of the shear stress ACFs in the atomic hydrodynamic representation, as well as their extrapolation in the low temperature regions. The correlation functions and consequently zero wave-vector viscosity follow the expected behavior when decreasing the temperature and the overall shape of the kernel at low temperatures is primarily affected by the attractive part of the potential. The data near and below the glass transition behave like a delta function in reciprocal space. Otherwise, it is well represented by a Lorentzian-type or two-term Gaussian function.

In the spatial domain and close to the glass transition temperature, the width of the kernel increases significantly from 4-6 atomic diameters at $T=1$ to at least 10 atomic diameters at $T=0.5$ followed by a dramatic increase closer to $T_{g}$. It is found that a dynamic scaling factor obtained from the reciprocal space viscosity kernels reduces the real space kernels to a unique form unlike the static scaling factors based on pair distribution functions. This is a further evidence that the slow dynamics in supercooled liquids is governed by a dynamic critical point at which time and length scales diverge. ${ }^{57}$

In conclusion, the response of polymer melts to a velocity gradient near $T_{g}$ turns out to be highly nonlocal. In systems where the strain rate varies significantly over these distances, the generalized viscosity must be used in order to correctly compute the velocity profile of polymer melts via use of generalized hydrodynamics. This implies that the classical Navier-Stokes hydrodynamics will fail for such systems. ${ }^{58}$

In view of the long runs required to obtain reliable data for the transport coefficients via equilibrium time correlation functions, the MD simulations should be extended to cover a much greater time range. ${ }^{59-61}$ We repeat that our conclusions are based on solid, but necessarily limited numerical evidence. Thus, we leave open the possibility that the zerowavevector zero-frequency viscosities change when a broader range of time scales is covered. However, we believe that the width of the real space kernels will not be significantly affected. In future work, it would also be interesting to study polymer melts in the entangled regime. ${ }^{27,62-64}$

\section{ACKNOWLEDGMENTS}

We thank the referees for their constructive comments and criticisms on the manuscript, which have greatly improved its quality.

${ }^{1}$ W. Götze and J. Sjögren, Rep. Prog. Phys. 55, 241 (1992).

${ }^{2}$ J. H. Gibbs and E. A. DiMarzio, J. Chem. Phys. 28, 373 (1958).

${ }^{3}$ G. S. Grest and M. Cohen, in Advances in Chemical Physics, edited by I. Prigogine and S. A. Rice (Wiley, New York, 1981), Vol. 48, pp. 455-525.

${ }^{4}$ W. Binder and J. Young, Rev. Mod. Phys. 58, 801 (1986).

${ }^{5}$ F. Varnik and K. Binder, J. Chem. Phys. 117, 6336 (2002).

${ }^{6}$ R. Yamamoto and A. Onuki, J. Chem. Phys. 117, 2359 (2002).

${ }^{7}$ K. Binder, J. Baschnagel, and W. Paul, Prog. Polym. Sci. 28, 115 (2003).

${ }^{8}$ M. L. Wallace, B. Joos, and M. Plischke, Phys. Rev. E 70, 041501 (2004).

${ }^{9}$ W. E. Alley and B. J. Alder, Phys. Rev. A 27, 3158 (1983). 
${ }^{10}$ D. J. Evans and G. P. Morriss, Statistical Mechanics of Nonequilibrium Liquids (Academic, New York, 1990).

${ }^{11}$ B. D. Todd, J. S. Hansen, and P. J. Daivis, Phys. Rev. Lett. 100, 195901 (2008).

${ }^{12}$ B. D. Todd and J. S. Hansen, Phys. Rev. E 78, 051202 (2008).

${ }^{13}$ J. S. Hansen, P. J. Daivis, K. P. Travis, and B. D. Todd, Phys. Rev. E 76, 041121 (2007).

${ }^{14}$ R. M. Puscasu, B. D. Todd, P. J. Daivis, and J. S. Hansen, J. Phys.: Condens. Matter 22, 195105 (2010).

${ }^{15}$ B. J. Palmer, Phys. Rev. E 49, 359 (1994).

${ }^{16}$ D. Bertolini and A. Tani, Phys. Rev. E 52, 1699 (1995).

${ }^{17}$ I. P. Omelyan, I. M. Mryglod, and M. V. Tokarchuk, Condens. Matter Phys. 8, 25 (2005).

${ }^{18}$ R. M. Puscasu, B. D. Todd, P. J. Daivis, and J. S. Hansen, Phys. Rev. E 82, 011801 (2010).

${ }^{19}$ A. Furukawa and H. Tanaka, Phys. Rev. Lett. 103, 135703 (2009).

${ }^{20}$ J. Kim and T. Keyes, J. Phys. Chem. 109, 21445 (2005).

${ }^{21}$ M. Isobe and B. J. Alder, Mol. Phys. 107, 609 (2009).

${ }^{22}$ J. H. Irving and J. G. Kirkwood, J. Chem. Phys. 18, 817 (1950).

${ }^{23}$ R. D. Olmsted and R. F. Snider, J. Chem. Phys. 65, 3407 (1976).

${ }^{24}$ B. D. Todd and P. J. Daivis, Mol. Simul. 33, 189 (2007).

${ }^{25}$ M. Doi, Introduction to Polymer Physics (Clarendon Press, Oxford, 1996).

${ }^{26}$ P. J. Daivis, M. L. Matin, and B. D. Todd, J. Non-Newtonian Fluid Mech. 111, 1 (2003).

${ }^{27}$ K. Kremer and G. S. Grest, J. Chem. Phys. 92, 5057 (1990).

${ }^{28}$ J. D. Weeks, D. Chandler, and H. C. Andersen, J. Chem. Phys. 54, 5237 (1971).

${ }^{29}$ K. Binder, C. Bennemann, J. Baschnagel, and W. Paul, in R. Kutner, A. Pekalski, and K. Sznajd-Weron, Anomalous Diffusion: From Basics to Applications (Springer, Berlin, 1999), pp. 124-139.

${ }^{30}$ G. B. Mc Kenna, in Comprehensive Polymer Science, edited by C. Booth and C. Proce, (Pergamon, Oxford, 1986), Vol. 2, pp. 311-362.

${ }^{31}$ P. J. Daivis, M. L. Matin, and B. D. Todd, J. Non-Newtonian Fluid Mech. 147, 35 (2007).

${ }^{32}$ M. Kröger, W. Loose, and S. Hess, J. Rheol. 37, 1057 (1993).

${ }^{33}$ C. W. Gear, The Numerical Integration of Ordinary Differential Equations of Various Orders (Argonne National Laboratory, Argonne, 1966).

${ }^{34}$ C. W. Gear, Numerical Initial Value Problems in Ordinary Differential Equations (Prentice-Hall, Englewood Cliffs, NJ, 1971).

${ }^{35}$ R. Edberg, D. J. Evans, and G. P. Morriss, J. Chem. Phys. 84, 6933 (1986).

${ }^{36}$ R. Edberg, D. J. Evans, and G. P. Morriss, Mol. Phys. 62, 1357 (1987).
${ }^{37}$ G. P. Morriss and D. J. Evans, Comput. Phys. Commun. 62, 267 (1991).

${ }^{38}$ M. P. Allen and D. J. Tildesley, Computer Simulation of Liquids (Clarendon, Oxford, 1989).

${ }^{39}$ T. A. Hunt and B. D. Todd, J. Chem. Phys. 131, 054904 (2009).

${ }^{40}$ R. M. Puscasu, Ph.D. thesis, Swinburne University of Technology, 2010.

${ }^{41}$ L. Berthier and T. A. Witten, Phys. Rev. E 80, 021502 (2009).

${ }^{42}$ L. Berthier and T. A. Witten, EPL 86, 10001 (2009).

${ }^{43}$ M. Rosche, R. G. Winkler, P. Reineker, and M. Schulz, J. Chem. Phys. 112, 3051 (2000)

${ }^{44}$ J. A. McCormick, C. K. Hall, and S. A. Khan, J. Chem. Phys. 122, 114902 (2005).

${ }^{45}$ C. Bennemann, W. Paul, K. Binder, and B. Dünweg, Phys. Rev. E 57, 843 (1998).

${ }^{46}$ M. Bulacu and E. Van der Giessen, Phys. Rev. E 76, 011807 (2007).

${ }^{47}$ M. Kröger and S. Hess, Phys. Rev. Lett. 85, 1128 (2000).

${ }^{48}$ T. A. Hunt and B. D. Todd, Mol. Simul. 35, 1153 (2009).

${ }^{49}$ R. Peck, C. Olsen, and J. Devore, Introduction to Statistics and Data Analysis (Brooks-Cole, Belmont, MA, 2008)

${ }^{50}$ L. Berthier, Phys. Rev. E 69, 020201 (2004).

${ }^{51}$ A. Papoulis, The Fourier Integral and Its Applications (McGraw-Hill, New York, 1962)

${ }^{52}$ L. Berthier and G. Tarjus, Phys. Rev. Lett. 103, 170601 (2009).

${ }^{53}$ M. M. Hurley and P. Harrowell, Phys. Rev. E 52, 1694 (1995).

${ }^{54}$ J. P. Garrahan and D. Chandler, Phys. Rev. Lett. 89, 035704 (2002).

${ }^{55}$ S. Whitelam, L. Berthier, and J. P. Garrahan, Phys. Rev. E 71, 026128 (2005).

${ }^{56}$ S. R. Williams and D. Evans, J. Chem. Phys. 132, 184105 (2010).

${ }^{57}$ S. Whitelam, L. Berthier, and J. P. Garrahan, Phys. Rev. Lett. 92, 185705 (2004).

${ }^{58}$ J. Goyon, A. Colin, G. Ovarlez, A. Ajdari, and L. Bocquet, Nature (London) 454, 84 (2008).

${ }^{59}$ S. Sen, S. K. Kumar, and P. Keblinski, J. Chem. Phys. 124, 144909 (2006).

${ }^{60}$ J. Ramírez, S. K. Sukumaran, and A. E. Likhtman, J. Chem. Phys. 126, 244904 (2007).

${ }^{61}$ J. Ramírez, S. K. Sukumaran, and A. E. Likhtman, Macromol. Symp. 252, 119 (2007).

${ }^{62}$ R. Yamamoto and A. Onuki, Phys. Rev. E 70, 041801 (2004).

${ }^{63}$ A. E. Likhtman, S. K. Sukumaran, and J. Ramirez, Macromolecules 40, 6748 (2007)

${ }^{64}$ D. J. Read, K. Jagannathan, and A. E. Likhtman, Macromolecules 41, $6843(2008)$. 